\title{
On positive solutions of a system of equations generated by Hadamard fractional operators
}

\author{
Amira M. Abdalla ${ }^{1 *}$, Hussein A.H. Salem ${ }^{1}$ and Kinga Cichoń ${ }^{2}$
}

\section{"Correspondence:}

amiramostafa@alexu.edu.eg

'Department of Mathematics and

Computer Science, Faculty of

Sciences, Alexandria University,

Alexandria, Egypt

Full list of author information is

available at the end of the article

\begin{abstract}
This paper is devoted to studying some systems of quadratic differential and integral equations with Hadamard-type fractional order integral operators. We concentrate on general growth conditions for functions generating right-hand side of considered systems, which leads to the study of Hadamard-type fractional operators on Orlicz spaces. Thus we need to prove some properties of such type of operators. In contrast to the case of Caputo or Riemann-Liouville type of fractional operators, it is not a convolution-type operator, so we need to study some of their new properties. Some more general problems than systems of quadratic integral equations are also studied, and the results are new even in the context of a single integral equation with the Hadamard fractional operator. The paper concludes with illustrative examples.
\end{abstract}

MSC: 26A33; 34A08

Keywords: Hadamard fractional integral; Quadratic equation; Orlicz space; Coupled system

\section{Introduction}

The study of quadratic differential and integral equations has a long history. They have been investigated by many authors. Initial studies were started by Chandrasekhar (see, e.g., $[4,13,19,28]$ and the references therein), and a beginning for this theory was mainly made by astrophysicists. Then research was conducted by mathematicians. They found some interesting open questions in this theory as well as many applications, for instance, in the theory of radiative transfer, kinetic theory of gases, and in the traffic theory.

However, over the last years, the theory of quadratic integral equations with nonsingular kernels has received a lot of attention (see, e.g., $[17,18,21,28,29]$ and the references therein). In most of the above-mentioned literature, the main results are realized with the help of the technique associated with the measures of noncompactness and a fixed point theorem of Darbo type. In this paper we study a singular problem, and we clarify that earlier approach seems to be too restrictive in our case.

On the other hand, different equations containing fractional derivatives or integrals also form a growing branch of mathematics and applied mathematics. In this paper we study

(c) The Author(s) 2020. This article is licensed under a Creative Commons Attribution 4.0 International License, which permits use, sharing, adaptation, distribution and reproduction in any medium or format, as long as you give appropriate credit to the original author(s) and the source, provide a link to the Creative Commons licence, and indicate if changes were made. The images or other third party material in this article are included in the article's Creative Commons licence, unless indicated otherwise in a credit line to the material. If material is not included in the article's Creative Commons licence and your intended use is not permitted by statutory regulation or exceeds the permitted use, you will need to obtain permission directly from the copyright holder. To view a copy of this licence, visit http://creativecommons.org/licenses/by/4.0/. 
some systems of quadratic fractional integral equations with fractional integrals taken in the Hadamard sense. The counterpart of our results with fractional Riemann-Liouville or Caputo was studied. We consider a more interesting case of the Hadamard fractional integral, which is not of a convolution type, and such kind of operators should be investigated independently of Riemann-Liouville operators. We wish to consider the most general case, i.e., when Hadamard fractional integral operators are acting on Orlicz spaces (instead of Lebesgue spaces), and we apply the obtained results for the study of systems of quadratic equations of fractional order.

Note that the aim of this paper is two-fold. On the one hand, we discuss the properties of Hadamard-type fractional integral operators acting on Orlicz spaces, which seems to be interesting in itself. On the other hand, we apply those results in order to ensure the existence of positive continuous solutions for the following coupled system of singular quadratic integral equations of fractional type:

$$
\begin{cases}x_{1}(t)=H_{1}(t, \bar{x}(t))+x_{2}(t) \mathfrak{J}^{\alpha_{1}} f_{1}(t, \bar{x}(t)) \frac{\eta_{1}(\bar{x}(t))}{\zeta_{1}(\bar{x}(t))}, & t \in[1, e], \alpha_{1}>0, \\ x_{2}(t)=H_{2}(t, \bar{x}(t))+x_{1}(t) \mathfrak{J}^{\alpha_{2}} f_{2}(t, \bar{x}(t)) \frac{\eta_{2}(\bar{x}(t))}{\zeta_{2}(\bar{x}(t))}, & t \in[1, e], \alpha_{2}>0 .\end{cases}
$$

Here, $\mathfrak{J}^{\alpha}$ stands the Hadamard fractional integral operator, where $\bar{x}=\left(x_{1}, x_{2}\right), f_{i}$ is in an appropriate Orlicz space. By "singularity" we mean the possibility that $\zeta_{i}(0,0)=0$ and the possibility that $\eta_{i}(0,0)$ being undefined are permitted. In fact, in our investigations, we assume that $\eta_{i}:(0,+\infty)^{2} \rightarrow[0,+\infty)$ and $\zeta_{i}:[0,+\infty)^{2} \rightarrow[0,+\infty), i=1,2$, are nonlinear, continuous, and nondecreasing functions. By placing appropriate conditions on $H_{i}$ and $f_{i}$, we use a fixed point theorem to prove the existence of a continuous solution $\bar{x}$ to problem (1). The obtained results are new in the context of singularity of the system of quadratic equations and of the Hadamard fractional operators with general growth conditions on Orlicz spaces.

We emphasize that much work on fractional differential and integral problems involves either Riemann-Liouville or Caputo type fractional differential equations. Another kind of fractional derivatives that appears side by side to Riemann-Liouville and Caputo derivatives in the literature is the fractional derivative due to Hadamard introduced in 1892 [23], which differs from the preceding ones in the sense that the kernel of the integral (in the definition of Hadamard derivative) contains logarithmic function of arbitrary exponent, i.e., being natural extension for the Hadamard formula

$$
\int_{a}^{x} \frac{d t_{1}}{t_{1}} \int_{a}^{t_{1}} \frac{d t_{2}}{t_{2}} \cdots \int_{a}^{t_{n-1}} \frac{d t_{n}}{t_{n}}=\frac{1}{\Gamma(n)} \int_{a}^{x}\left(\log \frac{x}{t}\right)^{n-1} \frac{f(t)}{t} d t
$$

with $n \in \mathbb{N}$ replaced by $\alpha \in \mathbb{R}^{+}$. One of its important advantages is that it is invariant with respect to dilatation on the whole axis, but surprisingly this kind of fractional calculus is still studied less than that of Riemann-Liouville.

It is worthwhile also to remark that the considered problem (1) has provoked some interest in earlier papers such as [30] and [31], but for fractional operators taken in the sense of Riemann-Liouville. However, the results of [30] and [31] cannot be applied in the case investigated here (cf. the miscellaneous examples introduced at the end of this paper). In our investigation, unlike, e.g., [17, 18, 21, 28, 29], no assumptions in terms of the measure of noncompactness were imposed on the nonlinearity of the given functions. For this 
reason, instead of the use of the technique associated with Darbo's fixed point theorem, we proceed in a different way by making use of the technique associated with Krasnoselskii's fixed point theorem for mappings with expanded cones. As the operator is not a contraction, we are unable to use the approach taken from [14].

Let us stress that our results are new even in the context of single quadratic fractional integral equations, in particular with the Hadamard fractional integrals. For an approach allowing to generalize growth conditions with the use of Orlicz spaces, see [14, Theorem 4.16] or [15, Remark 2], or [13, Theorem 5.1], but only for the case of quadratic integral equations with Riemann-Liouville integrals, or [16] for basics about Hadamard integral operators on Orlicz spaces.

\section{Preliminaries}

Throughout this paper by $C[a, b]$ we denote the space of continuous functions on the interval $[a, b]$, while $\mathcal{H}^{\lambda}[a, b]$ denotes the space of Hölder continuous functions of order $\lambda>0$. By $L_{p}[a, b], 1 \leq p \leq \infty$, we denote the Lebesgue space of real-valued measurable functions $f$ defined over $[a, b]$ such that $\int_{a}^{b}|f(s)| d s<\infty$, and by $L_{\infty}[a, b]$ we denote the Banach space of real-valued essentially bounded and measurable functions defined on $[a, b]$. We say that the pair $p, q \in[1, \infty]$ is of "conjugate exponents" if $p, q$ are connected by the relation $1 / p+1 / q=1$ for $1<p<\infty$ with the convention that $1 / \infty=0$. Some additional function spaces, such as Orlicz spaces, will be defined elsewhere.

Let us recall now some basics about cones and about our main tool, i.e., Krasnoselskii's fixed point theorem. The space $C:=C[a, b] \times C[a, b]$ equipped with the norm $\|\bar{x}\|_{\mathbb{R}_{2}}:=$ $\max \left\{\left\|x_{1}\right\|,\left\|x_{2}\right\|\right\}$ forms a Banach space. As it is a topological product of Banach spaces, by the Tychonoff theorem we are able to adapt the compactness criteria for $C$ taken from $C([a, b])$.

Recall that a cone $Q$ is a proper subset of $C$ such that if $u, v \in Q$ and $\lambda \in \mathbb{R}^{+}$, then $u+v$, $\lambda u \in Q$. Throughout this paper we consider closed convex $Q$ and its interior $Q^{\circ}$. This cone induces an order on $C$ defined by

1. $u \leq v$ if $v-u \in Q$,

2. $u<v$ if $v-u \in Q^{\circ}$.

A cone induces a partial order " $\geq$ " in $C$ by $x \geq y$ if $x-y \in Q$. In addition, we say that a function $\theta$ is nondecreasing on $Q$ if $\theta(x) \geq \theta(y)$ for all $x, y \in Q$ with $x \geq y$. A complete theory of fixed point theorems in ordered Banach spaces can be found in [10].

The following fixed point theorem on a cone expansion is due to Krasnoselskii (1960).

Theorem 1 ([37, Theorem 2.13]) Let $Q$ be a cone and $(C, Q)$ be an ordered Banach space. Let $Q_{1}$ and $Q_{2}$ be open subsets of $C$ with $\theta \in Q_{1}$ and $\bar{Q}_{1} \subset Q_{2}$.

Suppose that the operator $T: Q \cap\left(\bar{Q}_{2} \backslash Q_{1}\right) \rightarrow Q$ is continuous and compact. Further, suppose that it is a cone compression or expansion, i.e., either

1- $\|T(x)\| \leq\|x\|$ for $x \in Q \cap \partial Q_{1}$ and $\|T(x)\| \geq\|x\|$ for $x \in Q \cap \partial Q_{2}$, or

2- $\|T(x)\| \geq\|x\|$ for $x \in Q \cap \partial Q_{1}$ and $\|T(x)\| \leq\|x\|$ for $x \in Q \cap \partial Q_{2}$.

Then $T$ has a fixed point in $Q \cap\left(\bar{Q}_{2} \backslash Q_{1}\right)$.

Let us mention here that if $Q_{1}$ and $Q_{1}$ denote the balls with radii $r_{1}$ and $r_{2}$ (say $r_{1}<r_{2}$ ), respectively, then the above condition reads as follows:

$1^{0}\|T(x)\| \leq\|x\|$ for $\|x\|=r_{1}$ and $\|T(x)\| \geq\|x\|$ for $\|x\|=r_{2}$, or 
$2^{0}\|T(x)\| \geq\|x\|$ for $\|x\|=r_{1}$ and $\|T(x)\| \leq\|x\|$ for $\|x\|=r_{2}$.

In such a case the fixed point $x$ of $T$ exists with $r_{1}<\|x\|<r_{2}$. For our goals, this version is sufficient. For a detailed theory of fixed points on abstract cones, see [22].

\subsection{Hadamard-type operators}

Now, we are in a position to introduce the definition of the Hadamard-type fractional integral operator.

Definition 1 The Hadamard-type fractional integral of a given function $f$ of order $\alpha>0$ with left-hand point $a$ is defined by

$$
\mathfrak{J}_{a}^{\alpha} f(t):=\frac{1}{\Gamma(\alpha)} \int_{a}^{t}\left(\log \frac{t}{s}\right)^{\alpha-1} \frac{f(s)}{s} d s, \quad 0<a<t, \alpha>0
$$

provided that this integral exists. For complementation, we define $\mathfrak{J}_{a}^{\alpha} f(a):=0$. If it does not cause misunderstanding, then let us simplify and write $\mathfrak{J}_{a}^{\alpha}=\mathfrak{J}^{\alpha}$.

If not stated otherwise, we will assume that $\alpha \in(0,1)$. However, the above definition and some other results are presented with the most general assumption about $\alpha$.

One of the goals of the paper is to prove some useful properties of such operators. To do this, let us first recall some motivations for the study of such kind of operators and their known properties. Next, some new properties will be proved.

At least for the last 30 years, many authors have been carrying out research in development of the field of Riemann-Liouville fractional calculus (see [2, 3, 20, 26, 29, 35] and the references therein). However, as we mentioned earlier, it is not the only fractional calculus. Some basic properties of Hadamard fractional derivative and integral can be found in $[7,8,27]$ for instance. Although the Hadamard-type fractional calculus is an old topic, this type of fractional calculus has not yet been well studied and there is still much to explore. Let us only note some recent results in this direction $([1,7,8,25,36]$ or [24]). Related work on such operators in abstract spaces can be found in [32]. For more results about the fractional calculus in abstract spaces, we refer to $[16,34]$ and the references therein.

It is well known (see, e.g., $[26,35]$ ) that the operator $\mathfrak{J}_{a}^{\alpha}$ is defined on the space $L_{p}[a, b]$, $p \in[1, \infty]$. As a consequence of Hölder's inequality, it can be easily shown that the operator $\mathfrak{J}_{a}^{\alpha}$ maps $L_{p}[a, b]$ continuously into $L_{p}[a, b]$ for each $p \in[1, \infty]$.

Let us now investigate this operator on a larger class of spaces, namely on Orlicz spaces. We need to recall some necessary notions.

A function $M:[0,+\infty) \rightarrow[0,+\infty)$ is called a Young function if it has the form

$$
M(u)=\int_{0}^{u} a(s) d u \quad \text { for } u \geq 0,
$$

where $a:[0,+\infty) \rightarrow[0,+\infty)$ is an increasing, left-continuous function which is neither identically zero nor identically infinite on $[0,+\infty)$. In particular, if $M$ is finite-valued, where $\lim _{u \rightarrow 0} \frac{M(u)}{u}=0, \lim _{u \rightarrow \infty} \frac{M(u)}{u}=\infty$, and $M(u)>0$ if $x>0(M(u)=0 \Longleftrightarrow u=0)$, then $M$ is called an $N$-function.

The functions $M$ and $N$ are called complementary Young functions if

$$
N(x)=\sup _{y \geq 0}(x y-M(x)) .
$$


The Orlicz class, denoted by $\mathcal{O}_{P}$, consists of measurable functions $x: I \rightarrow \mathbb{R}$ for which

$$
\rho(x ; M)=\int_{I} M(x(t)) d t<\infty .
$$

We shall denote by $L_{M}(I)$ the Orlicz space of all measurable functions $x: I \rightarrow \mathbb{R}$ for which

$$
\|x\|_{M}=\inf _{\lambda>0}\left\{\int_{I} M\left(\frac{x(s)}{\lambda}\right) d s \leq 1\right\} .
$$

As the Young function $M(u)=\frac{|u|^{p}}{p}, 1<p<\infty$, leads to the classical Lebesgue space $L_{p}[a, b]$ with the norm mentioned before, we can treat an approach via Orlicz spaces as more general than the previous ones. Moreover, we should mention that for Young functions $M$ and $P$ the inclusion $L_{M} \subset L_{P}$ holds if and only if there exist positive constants $u_{0}$ and $a$ such that $P(u) \leq a M(u)$ for $u \geq u_{0}$. The last comment indicates that, in contrast to the case of Lebesgue spaces, we have no linearity of inclusions of spaces with respect to generating functions, and we should be very careful by investigating the set of values of operators.

We need to recall a deep result extending the known case of Lebesgue spaces, which is the main tool in carrying out our investigations.

Lemma 1 ([16, Theorem 2]) If $\alpha \in(0,1]$, for any Young function $\psi$ with its complementary function $\tilde{\psi}$ such that

$$
\int_{0}^{t} \tilde{\psi}\left(s^{\alpha-1}\right) d s<\infty, \quad t \in[1, e]
$$

the operator $\mathfrak{J}^{\alpha}$ is continuous from the Orlicz space $L_{\psi}([1, e])$ into $C[1, e]$.

Examples of such Young functions satisfying (3) are natural and can be found in [16, Remark 1, Example 1]. In particular, under such conditions $\mathfrak{J}^{\alpha}: C[1, e] \rightarrow C[1, e]$, let us present an auxiliary important proposition.

Proposition $1([16,33])$ Let $t>0, \alpha \in(0,1)$. For any Young function $\psi$, the function $\Psi$ : $[0, \infty) \rightarrow[0, \infty)$ defined by

$$
\Psi(t):=\left\{k>0: \int_{0}^{t k \frac{1}{1-\alpha}} \psi\left(s^{\alpha-1}\right) d s \leq k^{\frac{1}{1-\alpha}}\right\}
$$

is increasing and continuous with $\Psi(0)=0$.

Now a comment about quadratic operators. Let $H$ be a quadratic-type fractional operator of the form $H=F \cdot \mathfrak{J}^{\alpha}$ with the pointwise product of operators.

Lemma 2 If $F: C[1, e] \rightarrow C[1, e]$ is continuous and a Young function $\psi$ satisfies (3) and if $F: C \rightarrow C$ is continuous, then the operator $H=F \cdot \mathfrak{J}^{\alpha}$ maps $C[1, e]$ into itself and is continuous. 
Proof In view of Lemma 1 we get the continuity of $\mathfrak{J}^{\alpha}$ when it acts between $L_{\psi}[1, e]$ and $C[1, e]$, so it is true also for $\mathfrak{J}^{\alpha}: C[1, e] \rightarrow C[1, e]$ as this space is a Banach algebra with the pointwise product and $\|H\|_{\infty} \leq\|F\|_{\infty} \cdot\left\|\mathfrak{J}^{\alpha}\right\|_{\infty}$ and we are done.

It is a general result, parallel to that for weakly singular operators investigated in [14], so it can be interesting in itself. Usually, the most interesting case is when $F$ is the Nemytskii superposition operator (see classical quadratic equations). Another interesting case is when both operators are of fractional type. We will apply it (see Theorem 2 for instance) for a special case $F(x)=x$.

We should note that a special case covered by the above lemma is the case of power operators (cf. [9, Theorem 3.2, Remark 3] or [5]. Such operators decrease the norm for a small argument and increase for a sufficiently big one, so they do not complicate too much the idea of the proof for quadratic problems. For instance, such operators do not destroy assumptions of Theorem 1 when we multiply them by fractional integral operators, as $\left|\frac{1}{\gamma^{(n-1)}} \cdot x^{n}\right| \geq|x|$ for $x \geq \gamma>0$ and $\left|\frac{1}{\gamma^{(n-1)}} \cdot x^{n}\right| \leq|x|$ whenever $x \leq \gamma$ (see [9] for more examples). We propose to study such "generalized quadratic" problems as corollaries of the above lemmas together with quadratic ones.

More generally, for the case of integral equations, we should not investigate differentiability properties of solutions, so they are expected outside of the space of continuous functions (in $L_{p}$ or $L_{\varphi}$ spaces for instance). However, in the case of quadratic problems it causes problems. The key point is to ensure that the pointwise product is in the expected space of solutions (see $[12,13,15]$ for instance). Thus, by studying quadratic fractional Hadamard-type integral equations in function spaces, we will need the following.

The most general result which is based on Lemma 1 can be formulated as follows.

Lemma 3 Let $\psi$ be a Young function satisfying (3). Assume that a function space $X \subset$ $L_{\psi}([1, e])$ is such that its space of pointwise multipliers $M_{p}(X)$ contains the space $C[1, e]$ and that $F: X \rightarrow X$ is continuous. Then the operator $H=F \cdot \mathfrak{J}^{\alpha}$ maps $X$ into itself and is continuous.

The proof is straightforward, it suffices to apply the following multipliers property: $\|H\|_{X} \leq\|F\|_{X} \cdot\left\|\mathfrak{J}^{\alpha}\right\|_{\infty}$ (cf. [14]) and follow the lines of the proof of Lemma 1. Despite the fact that the assumption seems to be not really restrictive and many function spaces satisfy it, not every function space has the expected property (even for Banach algebras, cf. the space of functions with bounded variation $B V([1, e])$ for instance). We will show how useful this version is, too.

Let $\psi$ be a Young function with a complementary function $\tilde{\psi}$ satisfying (3). Let us fix the following set of assumptions on $f$ for the remaining part of the paper:

(1) $f:[1, e] \times \mathbb{R}^{2} \rightarrow \mathbb{R}$ is a Carathéodory function on $[1, e] \times \mathbb{R}^{2}$, i.e., for any $\bar{x} \in \mathbb{R}^{2}$, the function $f(\cdot, \bar{x})$ is measurable on $[1, e]$, and for almost every $t \in[1, e], f(t, \cdot)$ is continuous on $\mathbb{R}^{2}$ ),

(2) For any $\gamma>0$, there exists $M_{\gamma} \in L_{\psi}([1, e])$ with positive values such that $|f(t, \bar{x})| \leq M_{\gamma}(t), t \in[1, e]$ and $\|\bar{x}\| \leq \gamma$.

It means that we are ready to investigate our quadratic problem under general growth conditions on $f$. In fact, it allows us to investigate the Nemytskii superposition operator generated by $f$ as acting on $C[1, e]$ with values in $L_{\psi}([1, e])$ and, in view of the above results, 
the Hadamard fractional integral operator should have its values again in $C[1, e]$. It helps us to study the problem under more general assumptions than in the earlier mentioned papers. Let us state some immediate consequences of our assumptions.

Lemma 4 Iff $:[1, e] \times \mathbb{R} \rightarrow \mathbb{R}$ satisfies assumptions (1)-(2), then

1. The function $M_{\gamma}(t)$ satisfies $M_{\gamma}(t) \geq \max _{\|\bar{x}\| \leq \gamma}|f(t, \bar{x})|, \gamma>0, t \in[1, e]$;

2. For any $\bar{x} \in C[1, e], f(\cdot, \bar{x}(\cdot)) \in L_{\psi}([1, e])$;

3. The following inequality holds true: $\|f(\cdot, \bar{x}(\cdot))\|_{\psi} \leq\left\|M_{\gamma}\right\|_{\psi}$.

Proof The first claim is immediate. For the second and third, let us observe that under assumption (1) for any $\bar{x} \in C$ the superposition $N_{f}(\bar{x})=f(\cdot, \bar{x}(\cdot))$ is measurable and for any fixed $\gamma>0$ by (2) $\left\|N_{f}(\bar{x})\right\|_{\psi} \leq\left\|M_{\gamma}\right\|_{\psi}$, so we are done.

\section{System of Hadamard-type fractional integral equations}

In this section, we employ some consequences of Lemma 1 in order to assure the existence of positive continuous solutions for system (1). Recall that the singularity of equations is allowed, so both $\eta_{i}(0,0)$ can be undefined and the possibility that $\zeta_{i}(0,0)=0$ is allowed.

To facilitate our discussion, let us fix the following assumptions:

1. Assume that, for $i=1,2$, functions $f_{i}:[1, e] \times[0, \infty)^{2} \rightarrow(0, \infty)$ satisfy assumptions (1)-(2).

2. For $i=1,2$, functions $\eta_{i}:(0, \infty)^{2} \rightarrow[0, \infty)$ are continuous and nondecreasing with respect to the ordering in $\mathbb{R}^{2}$, that is, $\eta_{i}\left(x_{1}, x_{2}\right) \leq \eta_{i}\left(y_{1}, y_{2}\right)$ whenever $x_{j}<y_{j}, j=1,2$.

3. For $i=1,2$, functions $\zeta_{i}:[0, \infty)^{2} \rightarrow[0, \infty)$ are continuous and nondecreasing with respect to the ordering in $\mathbb{R}^{2}$ such that $\zeta_{i}\left(x_{1}, x_{2}\right)>0$ for all $\left(x_{1}, x_{2}\right) \neq(0,0)$.

4. For $i=1,2$, functions $H_{i}:[1, e] \times[0, \infty)^{2} \rightarrow(0, \infty)$ are continuous and satisfy the following conditions:

(a) $0<\mu_{i}<\gamma_{i}, i=1,2$, exist so that

$$
\text { for any } t \in[1, e], \mu_{i} \leq H_{i}(t, \bar{x}) \text { holds for every } \bar{x} \leq \bar{\mu} \text { and } \gamma_{i} \geq 2 \max _{t \in[1, e]} H_{i}(t, \bar{\mu}) \text {, }
$$

where $\bar{x}=\left(x_{1}, x_{2}\right)$ and $\bar{\mu}=\left(\mu_{1}, \mu_{2}\right)$.

(b) For $i=1,2$, there exist a function $b \in C[1, e]$ and nondecreasing functions $c_{i j}:\left[\mu_{i}, \infty\right) \rightarrow \mathbb{R}^{+}, j=1,2$, such that

$$
\left|H_{i}(t, \bar{x})-H_{i}(s, \bar{y})\right| \leq c_{i 1}\left(\gamma_{i}\right)|b(t)-b(s)|+c_{i 2}\left(\gamma_{i}\right)\|\bar{x}-\bar{y}\|_{\mathbb{R}^{2}}
$$

for all $\bar{x}, \bar{y} \in \mathbb{R}^{2}, x_{i}, y_{i} \in\left[\mu_{i}, \gamma_{i}\right]$, and $t, s \in[1, e]$.

Now, we are prepared to formulate and prove the existence result of our quadratic problem in the space of continuous functions. The next theorem deals with some conditions ensuring the existence of positive continuous bounded solutions for system (1).

Theorem 2 Let $\alpha_{1}, \alpha_{2} \in(0,1]$. Assume that $M_{i} \in L_{\psi}([1, e],(0, \infty)), i=1,2$, where $\psi$ is an appropriate Young function with its complementary function $\tilde{\psi}$ satisfying

$$
\int_{0}^{t} \tilde{\psi}\left(s^{\alpha-1}\right) d s<\infty, \quad t \in[1, e], \text { where } \alpha:=\max _{i}\left\{\alpha_{i}\right\}
$$


If assumptions (1.)-(4.) hold true along with

$$
\left(C_{2}(\gamma)+\frac{2 \tilde{\Psi}(1) \max _{i}\left\|M_{i}\right\|_{\psi}}{\Gamma(\alpha)} \max _{i}\left(\frac{\eta_{i}(\bar{\gamma})}{\zeta_{i}(\bar{\mu})}\right)\right) \leq \frac{1}{2},
$$

where $\gamma:=\max \left\{\gamma_{1}, \gamma_{2}\right\}, \beta:=\min \left\{\alpha_{1}, \alpha_{2}\right\}$, and $C_{2}(\gamma)=\max \left\{c_{12}\left(\gamma_{1}\right), c_{22}\left(\gamma_{2}\right)\right\}$, then system (1) possesses at least one solution $\bar{x} \in C$ such that $\bar{\mu} \leq \bar{x}(t) \leq \bar{\gamma}, t \in[1, e]$.

Proof To solve system (1), it is sufficient to find a fixed point of the operator $T: C \rightarrow C$ defined by

$$
T \bar{x}(t):=\left\{T_{1}\left(\bar{x}(t), T_{2}(\bar{x})(t)\right\}, \quad T_{i}: C \rightarrow C[1, e], i=1,2,\right.
$$

where

$$
T_{1}(\bar{x})(t):=H_{1}(t, \bar{x}(t))+\frac{x_{2}(t)}{\Gamma\left(\alpha_{1}\right)} \int_{1}^{t}\left(\log \frac{t}{s}\right)^{\alpha_{1}-1} f_{1}(s, \bar{x}(s)) \frac{\eta_{1}^{\star}(\bar{x}(s))}{\zeta_{1}^{\star}(\bar{x}(s))} \frac{d s}{s}, \quad t \in[1, e],
$$

and

$$
T_{2}(\bar{x})(t):=H_{2}(t, \bar{x}(t))+\frac{x_{1}(t)}{\Gamma\left(\alpha_{2}\right)} \int_{1}^{t}\left(\log \frac{t}{s}\right)^{\alpha_{2}-1} f_{2}(s, \bar{x}(s)) \frac{\eta_{2}^{\star}(\bar{x}(s))}{\zeta_{2}^{\star}(\bar{x}(s))} \frac{d s}{s}, \quad t \in[1, e] .
$$

Here, $\eta_{i}^{\star}: \mathbb{R}^{2} \rightarrow\left[\eta_{i}(\bar{\mu}), \eta_{i}(\bar{\gamma})\right]$ and $\zeta_{i}^{\star}: \mathbb{R}^{2} \rightarrow\left[\zeta_{i}(\bar{\mu}), \zeta_{i}(\bar{\gamma})\right](i=1,2)$ are defined by

$$
\eta_{i}^{\star}(\bar{x}):=\left\{\begin{array}{ll}
\eta_{i}(\bar{\gamma}) & \bar{x} \geq \bar{\gamma}, \\
\eta_{i}(\bar{x}) & \bar{\mu} \leq \bar{x} \leq \bar{\gamma}, \\
\eta_{i}(\bar{\mu}) & \bar{x} \leq \bar{\mu}
\end{array} \quad \text { and } \quad \zeta_{i}^{\star}(\bar{x}):= \begin{cases}\zeta_{i}(\bar{\gamma}) & \bar{x} \geq \bar{\gamma}, \\
\zeta_{i}(\bar{x}) & \bar{\mu} \leq \bar{x} \leq \bar{\gamma} \\
\zeta_{i}(\bar{\mu}) & \bar{x} \leq \bar{\mu} .\end{cases}\right.
$$

First we need to recall that $T$ is well defined on $C$. Observe that, for each $\bar{x} \in C$, functions $\frac{\eta_{i}^{\star}(\bar{x}(\cdot))}{\zeta_{i}^{*}(\bar{x} \cdot)}, i=1,2$, are continuous on $[1, e]$ and for any $\bar{x} \in C, f_{i}(\cdot, \bar{x}(\cdot))=f_{i}\left(\cdot, x_{1}(\cdot), x_{2}(\cdot)\right) \in$ $L_{\psi}([1, e])$.

Since $f_{i}(\cdot, \bar{x}(\cdot)) \frac{\eta_{i}^{\star}(\bar{x}(\cdot))}{\zeta_{i}^{*}(\cdot(\cdot))} \in L_{\psi}([1, e])$, by applying [16, Proposition 1] condition (6) holds true for both $\alpha_{1}, \alpha_{2}$, and in view of Lemma 1 it follows that the operator $T: C \rightarrow C$ is well defined.

Next, let us define the following subsets of the space $C$ (as required in Krasnoselskii's fixed point theorem):

$$
\begin{aligned}
& Q_{1}:=\left\{\bar{x} \in C:\|\bar{x}\|_{\mathbb{R}_{2}}<\mu:=\min \left\{\mu_{1}, \mu_{2}\right\}\right\} \text { and } \\
& Q_{2}:=\left\{\bar{x} \in C:\|\bar{x}\|_{\mathbb{R}_{2}}<\gamma:=\max \left\{\gamma_{1}, \gamma_{2}\right\}\right\}, \\
& Q:=\left\{\bar{x} \in C: x_{i}(t) \geq 0,\|\bar{x}(t)-\bar{x}(s)\|_{\mathbb{R}^{2}} \leq\left[\frac{B(t, s)+K \tilde{\Psi}(|t-s|)}{1-K^{*}}\right], t, s \in[1, e]\right\},
\end{aligned}
$$

where

$$
\begin{aligned}
& B(t, s):=C_{1}(\gamma)|b(t)-b(s)| \text { with } C_{1}(\gamma):=\max \left\{c_{11}\left(\gamma_{1}\right), c_{21}\left(\gamma_{2}\right)\right\}, \\
& K:=\frac{4 \gamma \max _{i}\left\|M_{i}\right\|_{\psi}}{\Gamma(\alpha)} \max _{i} \cdot\left\{\frac{\eta_{i}(\bar{\gamma})}{\zeta_{i}(\bar{\mu})}\right\},
\end{aligned}
$$


and

$$
K^{*}:=\left(C_{2}(\gamma)+\frac{2 \tilde{\Psi}(1) \max _{i}\left\|M_{i}\right\|_{\psi}}{\Gamma(\alpha)} \cdot \max _{i}\left\{\frac{\eta_{i}(\bar{\gamma})}{\zeta_{i}(\bar{\mu})}\right\}\right)<\frac{1}{2} .
$$

We divide our proof into five steps by proving some of important properties of $T$ :

(Step 1): $T: Q \cap\left(\bar{Q}_{2} \backslash Q_{1}\right) \rightarrow Q$ is well defined.

(Step 2): $T: Q \cap\left(\bar{Q}_{2} \backslash Q_{1}\right) \rightarrow Q$ is continuous.

(Step 3): $T: Q \cap\left(\bar{Q}_{2} \backslash Q_{1}\right) \rightarrow Q$ is compact.

(Step 4): $T$ satisfies a Krasnoselskii cone expansion/compression condition.

(Step 5): Every fixed point of $T$ solves system (1).

To prove the assertion of (Step 1$)$, let $\bar{x} \in Q \cap\left(\bar{Q}_{2} \backslash Q_{1}\right)$ and $t_{1}, t_{2} \in[a, b]$. With no loss of generality, we may assume that $t_{1} \leq t_{2}$ and then, keeping our assumption in mind, we have the following estimates:

$$
\begin{aligned}
& \left|T_{1}(\bar{x})\left(t_{2}\right)-T_{1}(\bar{x})\left(t_{1}\right)\right| \\
& \leq\left|H_{1}\left(t_{2}, \bar{x}\left(t_{2}\right)\right)-H_{1}\left(t_{1}, \bar{x}\left(t_{1}\right)\right)\right| \\
& +\mid \frac{x_{2}\left(t_{2}\right)}{\Gamma\left(\alpha_{1}\right)} \int_{1}^{t_{2}}\left(\log \frac{t_{2}}{s}\right)^{\alpha_{1}-1} f_{1}(s, \bar{x}(s)) \frac{\eta_{1}^{\star}(\bar{x}(s))}{\zeta_{1}^{\star}(\bar{x}(s))} \frac{d s}{s} \\
& -\frac{x_{2}\left(t_{1}\right)}{\Gamma\left(\alpha_{1}\right)} \int_{1}^{t_{1}}\left(\log \frac{t_{1}}{s}\right)^{\alpha_{1}-1} f_{1}(s, \bar{x}(s)) \frac{\eta_{1}^{\star}(\bar{x}(s))}{\zeta_{1}^{\star}(\bar{x}(s))} \frac{d s}{s} \\
& \leq c_{11}(\gamma)\left|b\left(t_{2}\right)-b\left(t_{1}\right)\right|+c_{12}(\gamma)\left\|\bar{x}\left(t_{2}\right)-\bar{x}\left(t_{1}\right)\right\|_{\mathbb{R}^{2}} \\
& +\mid \frac{x_{2}\left(t_{2}\right)}{\Gamma\left(\alpha_{1}\right)} \int_{1}^{t_{2}}\left(\log \frac{t_{2}}{s}\right)^{\alpha_{1}-1} f_{1}(s, \bar{x}(s)) \frac{\eta_{1}^{\star}(\bar{x}(s))}{\zeta_{1}^{\star}(\bar{x}(s))} \frac{d s}{s} \\
& -\frac{x_{2}\left(t_{1}\right)}{\Gamma\left(\alpha_{1}\right)} \int_{1}^{t_{2}}\left(\log \frac{t_{2}}{s}\right)^{\alpha_{1}-1} f_{1}(s, \bar{x}(s)) \frac{\eta_{1}^{\star}(\bar{x}(s))}{\zeta_{1}^{\star}(\bar{x}(s))} \frac{d s}{s} \mid \\
& +\mid \frac{x_{2}\left(t_{1}\right)}{\Gamma\left(\alpha_{1}\right)} \int_{1}^{t_{2}}\left(\log \frac{t_{2}}{s}\right)^{\alpha_{1}-1} f_{1}(s, \bar{x}(s)) \frac{\eta_{1}^{\star}(\bar{x}(s))}{\zeta_{1}^{\star}(\bar{x}(s))} \frac{d s}{s} \\
& -\frac{x_{2}\left(t_{1}\right)}{\Gamma\left(\alpha_{1}\right)} \int_{1}^{t_{1}}\left(\log \frac{t_{1}}{s}\right)^{\alpha_{1}-1} f_{1}(s, \bar{x}(s)) \frac{\eta_{1}^{\star}(\bar{x}(s))}{\zeta_{1}^{\star}(\bar{x}(s))} \frac{d s}{s} \\
& \leq c_{11}(\gamma)\left|b\left(t_{2}\right)-b\left(t_{1}\right)\right|+c_{12}(\gamma)\left\|\bar{x}\left(t_{2}\right)-\bar{x}\left(t_{1}\right)\right\|_{\mathbb{R}^{2}} \\
& +\frac{\left|x_{2}\left(t_{2}\right)-x_{2}\left(t_{1}\right)\right|}{\Gamma\left(\alpha_{1}\right)}\left(\frac{\eta_{1}(\bar{\gamma})}{\zeta_{1}(\bar{\mu})}\right) \int_{1}^{t_{2}}\left(\log \frac{t_{2}}{s}\right)^{\alpha_{1}-1}\left|f_{1}(s, \bar{x}(s))\right| \frac{d s}{s} \\
& +\frac{\left|x_{2}\left(t_{1}\right)\right|}{\Gamma\left(\alpha_{1}\right)}\left(\frac{\eta_{1}(\bar{\gamma})}{\zeta_{1}(\bar{\mu})}\right) \int_{t_{1}}^{t_{2}}\left(\log \frac{t_{2}}{s}\right)^{\alpha_{1}-1}\left|f_{1}(s, \bar{x}(s))\right| \frac{d s}{s} \\
& +\frac{\left|x_{2}\left(t_{1}\right)\right|}{\Gamma\left(\alpha_{1}\right)}\left(\frac{\eta_{1}(\bar{\gamma})}{\zeta_{1}(\bar{\mu})}\right) \int_{1}^{t_{1}}\left|\left(\log \frac{t_{2}}{s}\right)^{\alpha_{1}-1}-\left(\log \frac{t_{1}}{s}\right)^{\alpha_{1}-1}\right|\left|f_{1}(s, \bar{x}(s))\right| \frac{d s}{s} \\
& \leq c_{11}(\gamma)\left|b\left(t_{2}\right)-b\left(t_{1}\right)\right|+c_{12}(\gamma)\left\|\bar{x}\left(t_{2}\right)-\bar{x}\left(t_{1}\right)\right\|_{\mathbb{R}^{2}} \\
& +\frac{\left|x_{2}\left(t_{2}\right)-x_{2}\left(t_{1}\right)\right|}{\Gamma\left(\alpha_{1}\right)}\left(\frac{\eta_{1}(\bar{\gamma})}{\zeta_{1}(\bar{\mu})}\right) \int_{1}^{e} a_{1}(s)\left|M_{1}(s)\right| d s \\
& +\frac{\left|x_{2}\left(t_{1}\right)\right|}{\Gamma\left(\alpha_{1}\right)}\left(\frac{\eta_{1}(\bar{\gamma})}{\zeta_{1}(\bar{\mu})}\right) \int_{1}^{e}\left[a_{2}(s)+a_{3}(s)\right]\left|M_{1}(s)\right| d s,
\end{aligned}
$$


where

$$
a_{1}(s):=\left\{\begin{array}{ll}
\frac{\left(\log \frac{t_{2}}{s}\right)^{\alpha-1}}{s} & s \in\left[1, t_{2}\right], \\
0 & \text { otherwise }
\end{array} \quad \text { and } \quad a_{2}(s):= \begin{cases}\frac{\left(\log \frac{t_{2}}{s}\right)^{\alpha-1}}{s} & s \in\left[t_{1}, t_{2}\right], \\
0 & \text { otherwise }\end{cases}\right.
$$

and

$$
a_{3}(s):= \begin{cases}\frac{\left|\left(\log \frac{t_{2}}{s}\right)^{\alpha-1}-\left(\log \frac{t_{1}}{s}\right)^{\alpha-1}\right|}{s} & s \in\left[1, t_{1}\right] \\ 0 & \text { otherwise. }\end{cases}
$$

Arguing as in the proof of [16, Theorem 1], we ensure that $a_{i} \in L_{\tilde{\psi}}(i=1,2,3)$. Let us omit the details as they are similar to argumentations in the proof of [16, Theorem 2] with (small) necessary changes. In view of the Hölder inequality in Orlicz spaces, we conclude that

$$
\begin{aligned}
\mid T_{1}\left(\bar{x}\left(t_{2}\right)-T_{1}(\bar{x})\left(t_{1}\right) \mid \leq\right. & c_{11}(\gamma)\left|b\left(t_{2}\right)-b\left(t_{1}\right)\right|+c_{12}(\gamma)\left\|\bar{x}\left(t_{2}\right)-\bar{x}\left(t_{1}\right)\right\|_{\mathbb{R}^{2}} \\
& +\frac{\left|x_{2}\left(t_{2}\right)-x_{2}\left(t_{1}\right)\right|}{\Gamma\left(\alpha_{1}\right)}\left(\frac{\eta_{1}(\bar{\gamma})}{\zeta_{1}(\bar{\mu})}\right) \cdot 2\left\|a_{1}\right\|_{\tilde{\psi}}\left\|M_{1}\right\|_{\psi} \\
& +\frac{\left|x_{2}\left(t_{1}\right)\right|}{\Gamma\left(\alpha_{1}\right)}\left(\frac{f_{1}(\bar{\gamma})}{g_{1}(\bar{\mu})}\right) \cdot 2\left[\left\|a_{2}\right\|_{\tilde{\psi}}+\left\|a_{3}\right\|_{\tilde{\psi}}\right]\left\|M_{1}\right\|_{\psi} \\
\leq & c_{11}(\gamma)\left|b\left(t_{2}\right)-b\left(t_{1}\right)\right|+c_{12}(\gamma)\left\|\bar{x}\left(t_{2}\right)-\bar{x}\left(t_{1}\right)\right\|_{\mathbb{R}^{2}} \\
& +\frac{\left|x_{2}\left(t_{2}\right)-x_{2}\left(t_{1}\right)\right|}{\Gamma\left(\alpha_{1}\right)}\left(\frac{\eta_{1}(\bar{\gamma})}{\zeta_{1}(\bar{\mu})}\right) \cdot 2 \tilde{\Psi}\left(\log t_{2}\right)\left\|M_{1}\right\|_{\psi} \\
& +\frac{\left|x_{2}\left(t_{1}\right)\right|}{\Gamma\left(\alpha_{1}\right)}\left(\frac{\eta_{1}(\bar{\gamma})}{\zeta_{1}(\bar{\mu})}\right) \cdot 4 \tilde{\Psi}\left(\left|t_{2}-t_{1}\right|\right)\left\|M_{1}\right\|_{\psi} \\
\leq & C_{1}(\gamma)\left|b\left(t_{2}\right)-b\left(t_{1}\right)\right|+C_{2}(\gamma)\left\|\bar{x}\left(t_{2}\right)-\bar{x}\left(t_{1}\right)\right\|_{\mathbb{R}^{2}} \\
& +\frac{\left\|\bar{x}\left(t_{2}\right)-\bar{x}\left(t_{1}\right)\right\|_{\mathbb{R}^{2}}}{\Gamma(\alpha)} \max _{i}\left(\frac{\eta_{i}(\bar{\gamma})}{\zeta_{i}(\bar{\mu})}\right) \cdot 2 \tilde{\Psi}(1) \max _{i}\left\|M_{i}\right\|_{\psi} \\
& +\frac{4 \gamma}{\Gamma(\alpha)} \max _{i}\left(\frac{\eta_{i}(\bar{\gamma})}{\zeta_{i}(\bar{\mu})}\right) \cdot \tilde{\Psi}\left(\left|t_{2}-t_{1}\right|\right) \max _{i}\left\|M_{i}\right\|_{\psi} .
\end{aligned}
$$

Thus we conclude that $T_{1}(\bar{x}) \in C[1, e]$ :

$$
\left|T_{1}(\bar{x})\left(t_{2}\right)-T_{1}(\bar{x})\left(t_{1}\right)\right| \leq B\left(t_{1}, t_{2}\right)+K^{*}\left\|\bar{x}\left(t_{2}\right)-\bar{x}\left(t_{1}\right)\right\|_{\mathbb{R}^{2}}+K \tilde{\Psi}\left(\left|t_{2}-t_{1}\right|\right) .
$$

Similarly, the continuity for $T_{2}(\bar{x})$ on $[1, e]$ can be shown:

$$
\left|T_{2}(\bar{x})\left(t_{2}\right)-T_{2}(\bar{x})\left(t_{1}\right)\right| \leq B\left(t_{1}, t_{2}\right)+K^{*}\left\|\bar{x}\left(t_{2}\right)-\bar{x}\left(t_{1}\right)\right\|_{\mathbb{R}^{2}}+K \tilde{\Psi}\left(\left|t_{2}-t_{1}\right|\right) .
$$

Using the definition of $Q$, we get also the following estimation:

$$
\begin{aligned}
\left|T_{i}(\bar{x})\left(t_{2}\right)-T_{i}(\bar{x})\left(t_{1}\right)\right| & \leq B\left(t_{1}, t_{2}\right)+K^{*}\left[\frac{B(t, s)+K \tilde{\Psi}(|t-s|)}{1-K^{*}}\right]+K \tilde{\Psi}\left(\left|t_{2}-t_{1}\right|\right) . \\
& \leq \frac{1}{1-K^{*}}\left[B\left(t_{1}, t_{2}\right)+K \tilde{\Psi}\left(\left|t_{2}-t_{1}\right|\right)\right],
\end{aligned}
$$

which holds for every $i \in\{1,2\}$. Therefore, we conclude that $T(\bar{x}) \in Q$. 
As our assumptions imply that the values of $T_{1}, T_{2}$ are positive, i.e., $T_{i}(\bar{x})(t)>0, i=$ $1,2, t \in[1, e]$, it follows that $T(\bar{x}) \in Q$ for every $\bar{x} \in Q \cap\left(\bar{Q}_{2} \backslash Q_{1}\right)$, which implies that $T: Q \cap\left(\bar{Q}_{2} \backslash Q_{1}\right) \rightarrow Q$ and is well defined.

Next, we proceed to proving the assertion of (Step 2). To achieve this goal, we let $\bar{x}_{n} \rightarrow \bar{x}$ in $Q \cap\left(\bar{Q}_{2} \backslash Q_{1}\right)$, then $\bar{x}_{n}(t)=\left(x_{1, n}(t), x_{2, n}(t)\right) \rightarrow \bar{x}(t)=\left(x_{1}(t), x_{2}(t)\right)$ uniformly in $C$. In this case, with some further efforts one can get

$$
\begin{aligned}
& \left|T_{1}\left(\bar{x}_{n}\right)(t)-T_{1}\left(\bar{x}_{n}\right)(t)\right| \\
& \leq\left|H_{1}\left(t, \bar{x}_{n}(t)\right)-H_{1}(t, \bar{x}(t))\right| \\
& +\mid \frac{x_{2, n}(t)}{\Gamma\left(\alpha_{1}\right)} \int_{1}^{t}\left(\log \frac{t}{s}\right)^{\alpha_{1}-1} f_{1}\left(s, \bar{x}_{n}(s)\right) \frac{\eta_{1}^{\star}\left(\bar{x}_{n}(s)\right)}{\zeta_{1}^{\star}\left(\bar{x}_{n}(s)\right)} \frac{d s}{s} \\
& -\frac{x_{2}(t)}{\Gamma\left(\alpha_{i}\right)} \int_{1}^{t}\left(\log \frac{t}{s}\right)^{\alpha_{1}-1} f_{1}(s, \bar{x}(s)) \frac{\eta_{1}^{\star}(\bar{x}(s))}{\zeta_{1}^{\star}(\bar{x}(s))} \frac{d s}{s} \mid \\
& \leq c_{12}(\gamma)\left\|\bar{x}_{n}(t)-\bar{x}(t)\right\|_{\mathbb{R}^{2}} \\
& +\mid \frac{x_{2, n}(t)}{\Gamma\left(\alpha_{1}\right)} \int_{1}^{t}\left(\log \frac{t}{s}\right)^{\alpha_{1}-1} f_{1}\left(s, \bar{x}_{n}(s)\right) \frac{\eta_{1}^{\star}\left(\bar{x}_{n}(s)\right)}{\zeta_{1}^{\star}\left(\bar{x}_{n}(s)\right)} \frac{d s}{s} \\
& -\frac{x_{2}(t)}{\Gamma\left(\alpha_{i}\right)} \int_{1}^{t}\left(\log \frac{t}{s}\right)^{\alpha_{1}-1} f_{1}\left(s, \bar{x}_{n}(s)\right) \frac{\eta_{1}^{\star}\left(\bar{x}_{n}(s)\right)}{\zeta_{1}^{\star}\left(\bar{x}_{n}(s)\right)} \frac{d s}{s} \mid \\
& +\mid \frac{x_{2}(t)}{\Gamma\left(\alpha_{1}\right)} \int_{1}^{t}\left(\log \frac{t}{s}\right)^{\alpha_{1}-1} f_{1}\left(s, \bar{x}_{n}(s)\right) \frac{\eta_{1}^{\star}\left(\bar{x}_{n}(s)\right)}{\zeta_{1}^{\star}\left(\bar{x}_{n}(s)\right)} \frac{d s}{s} \\
& -\frac{x_{2}(t)}{\Gamma\left(\alpha_{1}\right)} \int_{1}^{t}\left(\log \frac{t}{s}\right)^{\alpha_{1}-1} f_{1}(s, \bar{x}(s)) \frac{\eta_{1}^{\star}\left(\bar{x}_{n}(s)\right)}{\zeta_{1}^{\star}\left(\bar{x}_{n}(s)\right)} \frac{d s}{s} \mid \\
& +\mid \frac{x_{2}(t)}{\Gamma\left(\alpha_{1}\right)} \int_{1}^{t}\left(\log \frac{t}{s}\right)^{\alpha_{1}-1} f_{1}(s, \bar{x}(s)) \frac{\eta_{1}^{\star}\left(\bar{x}_{n}(s)\right)}{\zeta_{1}^{\star}\left(\bar{x}_{n}(s)\right)} \frac{d s}{s} \\
& -\frac{x_{2}(t)}{\Gamma\left(\alpha_{1}\right)} \int_{1}^{t}\left(\log \frac{t}{s}\right)^{\alpha_{1}-1} f_{1}(s, \bar{x}(s)) \frac{\eta_{1}^{\star}(\bar{x}(s))}{\zeta_{1}^{\star}(\bar{x}(s))} \frac{d s}{s} \mid \\
& \leq c_{12}(\gamma)\left\|\bar{x}_{n}(t)-\bar{x}(t)\right\|_{\mathbb{R}^{2}} \\
& +\frac{\left|x_{2, n}(t)-x_{2}(t)\right|}{\Gamma\left(\alpha_{1}\right)} \frac{\eta_{1}(\bar{\gamma})}{\zeta_{1}(\bar{\mu})}\left(\max _{t \in[1, e]} \int_{1}^{t}\left(\log \frac{t}{s}\right)^{\alpha_{1}-1} M_{1}(s) \frac{d s}{s}\right) \\
& +\frac{\left|x_{2}(t)\right|}{\Gamma\left(\alpha_{1}\right)} \frac{\eta_{1}(\bar{\gamma})}{\zeta_{1}(\bar{\mu})} \int_{1}^{t}\left(\log \frac{t}{s}\right)^{\alpha_{1}-1}\left|f_{1}\left(s, \bar{x}_{n}(s)\right)-f_{1}(s, \bar{x}(s))\right| \frac{d s}{s} \\
& +\frac{\left|x_{2}(t)\right|}{\Gamma\left(\alpha_{1}\right)} \max _{t \in[1, e]}\left|\frac{\eta_{1}^{\star}\left(\bar{x}_{n}(t)\right)}{\zeta_{1}^{\star}\left(\bar{x}_{n}(t)\right)}-\frac{\eta_{1}^{\star}(\bar{x}(t))}{\zeta_{1}^{\star}(\bar{x}(t))}\right|\left(\max _{t \in[a, b]} \int_{a}^{t}\left(\log \frac{t}{s}\right)^{\alpha_{1}-1} M_{1}(s)\right) \frac{d s}{s} .
\end{aligned}
$$

Again by applying the Hölder inequality, we obtain

$$
\begin{aligned}
& \left|T_{1}\left(\bar{x}_{n}\right)(t)-T_{1}(\bar{x})(t)\right| \\
& \quad \leq c_{12}(\gamma)\left\|\bar{x}_{n}(t)-\bar{x}(t)\right\|_{\mathbb{R}^{2}} \\
& \quad+\frac{2 \tilde{\Psi}(1)\left\|M_{1}\right\|_{\psi}}{\Gamma\left(\alpha_{1}\right)} \frac{\eta_{1}(\bar{\gamma})}{\zeta_{1}(\bar{\mu})}\left\|\bar{x}_{n}(t)-\bar{x}(t)\right\|_{\mathbb{R}^{2}}
\end{aligned}
$$




$$
\begin{aligned}
& +\frac{\gamma}{\Gamma\left(\alpha_{1}\right)} \frac{\eta_{1}(\bar{\gamma})}{\zeta_{1}(\bar{\mu})} \int_{1}^{t}\left(\log \frac{t}{s}\right)^{\alpha_{1}-1}\left|f_{1}\left(s, \bar{x}_{n}(s)\right)-f_{1}(s, \bar{x}(s))\right| \frac{d s}{s} \\
& +\frac{2 \gamma \tilde{\Psi}(1)\left\|M_{1}\right\|_{\psi}}{\Gamma\left(\alpha_{1}\right)} \max _{t \in[1, e]}\left|\frac{\eta_{1}^{\star}\left(\bar{x}_{n}(t)\right)}{\zeta_{1}^{\star}\left(\bar{x}_{n}(t)\right)}-\frac{\eta_{1}^{\star}(\bar{x}(t))}{\zeta_{1}^{\star}(\bar{x}(t))}\right| \cdot \\
& \leq\left\|\bar{x}_{n}(t)-\bar{x}(t)\right\|_{\mathbb{R}^{2}}\left(C_{2}(\gamma)+\frac{2 \tilde{\Psi}(1) \max _{i}\left\|M_{i}\right\|_{\psi}}{\Gamma(\alpha)} \cdot \max _{i}\left\{\frac{\eta_{i}(\bar{\gamma})}{\zeta_{i}(\bar{\mu})}\right\}\right) \\
& +\frac{\gamma}{\Gamma(\alpha)} \max _{i}\left(\frac{\eta_{i}(\bar{\gamma})}{\zeta_{i}(\bar{\mu})} \int_{1}^{t}\left(\log \frac{t}{s}\right)^{\alpha-1}\left|f_{i}\left(s, \bar{x}_{n}(s)\right)-f_{i}(s, \bar{x}(s))\right| \frac{d s}{s}\right) \\
& +\frac{2 \gamma \tilde{\Psi}(1) \max _{i}\left\|M_{i}\right\|_{\psi}}{\Gamma(\alpha)} \max _{i}\left(\max _{t \in[1, e]}\left|\frac{\eta_{i}^{\star}\left(\bar{x}_{n}(t)\right)}{\zeta_{i}^{\star}\left(\bar{x}_{n}(t)\right)}-\frac{\eta_{i}^{\star}(\bar{x}(t))}{\zeta_{i}^{\star}(\bar{x}(t))}\right|\right) .
\end{aligned}
$$

Similarly,

$$
\begin{aligned}
\left|T_{2}\left(\bar{x}_{n}\right)(t)-T_{2}(\bar{x})(t)\right| \leq & \left\|\bar{x}_{n}(t)-\bar{x}(t)\right\|_{\mathbb{R}^{2}}\left(C_{2}(\gamma)+\frac{2 \tilde{\Psi}(1) \max _{i}\left\|M_{i}\right\|_{\psi}}{\Gamma(\alpha)} \cdot \max _{i}\left\{\frac{\eta_{i}(\bar{\gamma})}{\zeta_{i}(\bar{\mu})}\right\}\right) \\
& +\frac{\gamma}{\Gamma(\alpha)} \max _{i}\left(\frac{\eta_{i}(\bar{\gamma})}{\zeta_{i}(\bar{\mu})} \int_{1}^{t}\left(\log \frac{t}{s}\right)^{\alpha-1}\left|f_{i}\left(s, \bar{x}_{n}(s)\right)-f_{i}(s, \bar{x}(s))\right| \frac{d s}{s}\right) \\
& +\frac{2 \gamma \tilde{\Psi}(1) \max _{i}\left\|M_{i}\right\|_{\psi}}{\Gamma(\alpha)} \max _{i}\left(\max _{t \in[1, e]}\left|\frac{\eta_{i}^{\star}\left(\bar{x}_{n}(t)\right)}{\zeta_{i}^{\star}\left(\bar{x}_{n}(t)\right)}-\frac{\eta_{i}^{\star}(\bar{x}(t))}{\zeta_{i}^{\star}(\bar{x}(t))}\right|\right) .
\end{aligned}
$$

It is equivalent to the following estimate:

$$
\begin{aligned}
\left\|T\left(\bar{x}_{n}\right)-T(\bar{x})\right\|_{\mathbb{R}_{2}}= & \max _{i}\left\{\max _{t \in[1, e]}\left|T_{i}\left(\bar{x}_{n}\right)(t)-T_{i}(\bar{x})(t)\right|\right\} \\
\leq & \frac{1}{2}\left\|\bar{x}_{n}(t)-\bar{x}(t)\right\|_{\mathbb{R}^{2}} \\
& +\frac{\gamma}{\Gamma(\alpha)} \max _{i}\left(\frac{\eta_{i}(\bar{\gamma})}{\zeta_{i}(\bar{\mu})} \int_{1}^{t}\left(\log \frac{t}{s}\right)^{\alpha-1}\left|f_{i}\left(s, \bar{x}_{n}(s)\right)-f_{i}(s, \bar{x}(s))\right| \frac{d s}{s}\right) \\
& +\frac{2 \gamma \tilde{\Psi}(1) \max _{i}\left\|M_{i}\right\|_{\psi}}{\Gamma(\alpha)} \max _{i}\left(\max _{t \in[1, e]}\left|\frac{\eta_{i}^{\star}\left(\bar{x}_{n}(t)\right)}{\zeta_{i}^{\star}\left(\bar{x}_{n}(t)\right)}-\frac{\eta_{i}^{\star}(\bar{x}(t))}{\zeta_{i}^{\star}(\bar{x}(t))}\right|\right) .
\end{aligned}
$$

It implies, in view of the continuity of $\frac{\eta_{i}^{\star}(\cdot)}{\zeta_{i}^{\star}(\cdot)}, i=1,2$, and $f_{i}(t, \cdot), t \in[1, e], i=1,2$, that the operator $T: Q \cap\left(\bar{Q}_{2} \backslash Q_{1}\right) \rightarrow Q$ is continuous. The second claim is established.

Now we need to prove the assertion of (Step 3). Let $M \subset Q \cap\left(\bar{Q}_{2} \backslash Q_{1}\right)$ be bounded. Note that, for any $\bar{x} \in M$, we have

$$
\begin{aligned}
\left|T_{1}(\bar{x})(t)\right| \leq & \left|H_{1}(t, \bar{x}(t))\right|+\frac{\left|x_{2}(t)\right|}{\Gamma\left(\alpha_{1}\right)}\left(\frac{\eta_{1}(\bar{\gamma})}{\zeta_{1}(\bar{\mu})}\right)\left(\max _{t \in[1, e]} \int_{1}^{t}\left(\log \frac{t}{s}\right)^{\alpha_{1}-1} f_{1}(s, \bar{x}(s)) \frac{d s}{s}\right) \\
\leq & \left|H_{1}(t, \bar{x}(t))-H_{1}(t, \bar{\mu})+H_{1}(t, \bar{\mu})\right| \\
& +\left|x_{2}(t)\right| \frac{2 \tilde{\Psi}(1)\left\|M_{1}\right\|_{\psi}}{\Gamma\left(\alpha_{1}\right)}\left(\frac{\eta_{1}(\bar{\gamma})}{\zeta_{1}(\bar{\mu})}\right) \\
\leq & \left|H_{1}(t, \bar{x}(t))-H_{1}(t, \bar{\mu})\right|+\left\|H_{1}(\cdot, \bar{\mu})\right\| \\
& +\left|x_{2}(t)\right| \frac{2 \tilde{\Psi}(1)\left\|M_{1}\right\|_{\psi}}{\Gamma\left(\alpha_{1}\right)}\left(\frac{\eta_{1}(\bar{\gamma})}{\zeta_{1}(\bar{\mu})}\right)
\end{aligned}
$$




$$
\begin{aligned}
\leq & C_{2}(\gamma)\|\bar{x}(t)-\bar{\mu}\|_{\mathbb{R}^{2}}+\left\|H_{1}(\cdot, \bar{\mu})\right\| \\
& +\gamma \frac{2 \tilde{\Psi}(1) \max _{i}\left\|M_{i}\right\|_{\psi}}{\Gamma(\alpha)} \max _{i}\left(\frac{\eta_{i}(\bar{\gamma})}{\zeta_{i}(\bar{\mu})}\right) .
\end{aligned}
$$

As we have a simple estimation

$$
\|\bar{x}(t)-\bar{\mu}\|_{\mathbb{R}^{2}}=\max \left\{\max _{t \in[a, b]}\left|x_{1}(t)-\mu_{2}\right|, \max _{t \in[a, b]}\left|x_{2}(t)-\mu_{1}\right|\right\} \leq|\gamma-\mu| \leq \gamma,
$$

then in view of inequality (7), we get

$$
\left|T_{1}(\bar{x})(t)\right| \leq\left\|H_{1}(\cdot, \bar{\mu})\right\|+\gamma \frac{1}{2} \leq \frac{\gamma_{1}}{2}+\frac{\gamma}{2} .
$$

Similarly,

$$
\left|T_{2}(\bar{x})(t)\right| \leq \frac{\gamma_{2}}{2}+\frac{\gamma}{2} .
$$

Since $\gamma=\max \left\{\gamma_{1}, \gamma_{2}\right\}$, we conclude that $\|T(\bar{x})\|_{\mathbb{R}_{2}}=\max _{i \in\{1,2\}}\left\{\left\|T_{1}(\bar{x})\right\|,\left\|T_{2}(\bar{x})\right\|\right\}<\gamma$. It implies the boundedness of the image $T(M)$. Moreover, from (13), we conclude the equicontinuity of the set $T(M)$.

Finally, the continuous operator $T$ maps bounded subsets of $Q \cap\left(\bar{Q}_{2} \backslash Q_{1}\right)$ into bounded equicontinuous subsets of $Q$. In view of Tychonoff and Arzelà-Ascoli theorems, we obtain compactness of the continuous operator $T$, as expected in the assertion of (Step 3).

To verify (Step 4), we need to evaluate the norm of $T(x)$ on boundaries of $\partial Q_{1}$ and $\partial Q_{2}$. As we already proved that for $\bar{x} \in Q \cap \partial Q_{2}$ we have $\|T(\bar{x})\|_{\mathbb{R}_{2}}<\gamma=\|\bar{x}\|_{\mathbb{R}_{2}}$, we will verify the remaining part of assumption [2-] in Theorem 1.

To do this, let us observe that $0<x_{i}(t) \leq \mu, t \in[1, e], i=1,2$, for any $\bar{x}=\left(x_{1}, x_{2}\right) \in Q \cap$ $\partial Q_{1}$. In this case, by applying the monotonicity properties of $\eta_{i}$ and $\zeta_{i}(i=1,2)$, we have

$$
T_{1}(\bar{x})(t) \geq H_{1}(t, \bar{x}(t))+\frac{x_{2}(t)}{\Gamma\left(\alpha_{1}\right)}\left(\frac{\eta_{1}^{\star}(0)}{\zeta_{1}^{\star}(\bar{\mu})}\right)\left(\int_{a}^{t}\left(\log \frac{t}{s}\right)^{\alpha_{1}-1} f_{i}(s, \bar{x}(s)) \frac{d s}{s}\right)
$$

for all $t \in[1, e]$.

In view of assumption (4.)(a), for chosen $\bar{x}$, we get $H_{1}(t, \bar{x}(t)) \geq \mu_{1}$. Moreover, assumptions (2.) and (3.) imply that $\left(\frac{\eta_{1}^{\star}(0)}{\xi_{1}^{\star}(\bar{\mu})}\right)>0$, and as the operator $\mathfrak{J}_{a}^{\alpha}$ has nonnegative values, we get $\left\|T_{1}(\bar{x})\right\| \geq H_{1}(t, \bar{x}(t)) \geq \mu_{1}$. Similarly, $\left\|T_{2}(\bar{x})\right\| \geq \mu_{2}$. Consequently, $\|T \bar{x}\|_{\mathbb{R}_{2}} \geq$ $\min \left\{\mu_{2}, \mu_{2}\right\}=\mu \geq\|\bar{x}\|_{\mathbb{R}_{2}}$.

All the hypotheses of Krasnoselskii's fixed point theorem are fulfilled. Therefore, we can conclude that the operator $T: Q \cap\left(\bar{Q}_{2} \backslash Q_{1}\right) \rightarrow Q$ has at least one fixed point $\bar{x}=\left(x_{1}, x_{2}\right) \in$ $Q \cap\left(\bar{Q}_{2} \backslash Q_{1}\right)$, being continuous on $[1, e]$. Meaning that there would exist $x_{i} \in C[1, e], i=$ 1,2 , with the property that $x_{i}(t) \in(0, \gamma], \forall t \in[1, e], i=1,2$, for which we have

$$
\begin{cases}x_{1}(t)=H_{1}(t, \bar{x}(t))+\frac{x_{2}(t)}{\Gamma\left(\alpha_{1}\right)} \int_{a}^{t}\left(\log \frac{t}{s}\right)^{\alpha_{1}-1} f_{1}(s, \bar{x}(s)) \frac{\eta_{1}^{\star}(\bar{x}(s))}{\zeta_{1}^{\star}(\bar{x}(s))} \frac{d s}{s}, & t \in[1, e], \\ x_{2}(t)=H_{2}(t, \bar{x}(t))+\frac{x_{1}(t)}{\Gamma\left(\alpha_{1}\right)} \int_{a}^{t}\left(\log \frac{t}{s}\right)^{\alpha_{2}-1} f_{2}(s, \bar{x}(s)) \frac{\eta_{2}^{\star}(\bar{x}(s))}{\xi_{2}^{\star}(\bar{x}(s))} \frac{d s}{s}, & t \in[1, e] .\end{cases}
$$

Finally, for (Step 5), looking at system (14), observe that

$$
x_{i}(t) \geq H_{i}(t, \bar{x}(t)) \text { hold for all } t \in[1, e], i=1,2 .
$$


We conclude that $\bar{x}(t) \geq(\mu, \mu), \forall t \in[1, e]$. Otherwise, in view of our assumptions imposed on $H_{1}, H_{2}$, we obtain that

$$
\mu_{i} \geq \mu>x_{i}(t) \geq H_{i}(t, \bar{x}(t)) \geq \mu_{i}, \quad t \in[1, e](i=1,2),
$$

which, of course, would lead to contradiction. Consequently, since $\bar{\gamma} \geq \bar{x}(t) \geq \bar{\mu}, t \in[a, b]$, it follows in view of (14) and by the definitions of $\eta_{i}^{\star}$ and $\zeta_{i}^{\star}$ that $\bar{x} \in Q \cap\left(\bar{Q}_{2} \backslash Q_{1}\right)$ solves system (1), which completes the proof.

Remark 1 Now let us show an immediate consequence of the above theorem if we replace Lemma 1 by more general Lemma 3. In particular, this extension is useful when we study $F\left(x_{1}, x_{2}\right)=\left(x_{2}^{2}, x_{1}^{2}\right)$, i.e., cubic equations (see [5] for instance) or $F\left(x_{1}, x_{2}\right)=\left(x_{2}^{n-1}, x_{1}^{n-1}\right)$, i.e., $n$th order ([9]) (both proved by a contraction method), but we are not restricted only to those cases. Note that in earlier papers only the approach via contraction mappings was used. It should be also stressed that the case of Hadamard fractional operators is more complicated than that of the convolution-type operators.

Consider the following generalized system of Hadamard-type fractional integral equations:

$$
\begin{cases}x_{1}(t)=H_{1}(t, \bar{x}(t))+F_{1}\left(x_{2}\right)(t) \cdot \mathfrak{J}^{\alpha_{1}} f_{1}(t, \bar{x}(t)) \frac{\eta_{1}(\bar{x}(t))}{\zeta_{1}(\bar{x}(t))}, & t \in[1, e], \alpha_{1}>0, \\ x_{2}(t)=H_{2}(t, \bar{x}(t))+F_{2}\left(x_{1}\right)(t) \cdot \mathfrak{J}^{\alpha_{2}} f_{2}(t, \bar{x}(t)) \frac{\eta_{2}(\bar{x}(t))}{\zeta_{2}(\bar{x}(t))}, & t \in[1, e], \alpha_{2}>0 .\end{cases}
$$

For $(x, \rho) \in C[1, e] \times(0, \infty)$, we denote by $\omega(x, \rho)$ the modulus of continuity of $x \in C[1, e]$, i.e.,

$$
\omega(x, \rho)=\sup \left\{\left|x\left(t_{1}\right)-x\left(t_{2}\right)\right|:\left(t_{1}, t_{2}\right) \in[1, e] \times[1, e],\left|t_{1}-t_{2}\right| \leq \rho\right\} .
$$

Further on, for any subset $M$ of $C[1, e]$, let us define (cf. [6])

$$
\omega(M, \rho)=\sup \{\omega(x, \rho): x \in M\}
$$

and

$$
\omega(M)=\lim _{\rho \rightarrow 0} \omega(M, \rho)
$$

So, a function $x$ admits $\omega$ as a modulus of continuity if and only if $|x(t)-x(s)| \leq \omega(x,|t-s|)$. Moreover, for any continuous function $X$, this modulus is continuous at zero: $\omega(x, r) \rightarrow 0$ as $r \rightarrow 0$.

Recall that, as we consider continuous functions on compact interval, they are uniformly continuous. A function turns out to be uniformly continuous if and only if it admits a modulus of continuity. For the case of quadratic problems, it is worthwhile to note that if $x$ and $y$ are bounded real-valued functions with moduli respectively $\omega_{1}$ and $\omega_{2}$, then the pointwise product $x \cdot y$ has the modulus of continuity $\|y\|_{\infty} \cdot \omega_{1}+\|x\|_{\infty} \cdot \omega_{2}$. We are ready to prove an extension for Theorem 2, which will be then illustrated in Example 3.1. 
Proposition 2 Let $\alpha_{1}, \alpha_{2} \in(0,1]$. Assume that $M_{i} \in L_{\psi}([1, e],(0, \infty)), i=1,2$, where $\psi$ is an appropriate Young function with its complementary function $\tilde{\psi}$ satisfying

$$
\int_{0}^{t} \tilde{\psi}\left(s^{\alpha-1}\right) d s<\infty, \quad t \in[1, e], \text { where } \alpha:=\max _{i}\left\{\alpha_{i}\right\}
$$

Assume that, for bounded and continuous mapping $F\left(x_{1}, x_{2}\right)=\left(F_{1}\left(x_{2}\right), F_{2}\left(x_{1}\right)\right): C \rightarrow C$, there exists a constant $M_{L}$ such that, for any subset $M$ in $B_{\gamma}$ and any $r>0$, we have

$$
\max \left\{\omega\left(F_{1}(M), r\right), \omega\left(F_{2}(M), r\right)\right\} \leq M_{L} \cdot \omega(M, r) .
$$

If assumptions (1.)-(4.) hold true along with

$$
\left(C_{2}(\gamma)+\frac{2 \cdot \tilde{\Psi}(1) \max _{i}\left\|M_{i}\right\|_{\psi}}{\Gamma(\alpha)} \max _{i}\left(\frac{\eta_{i}(\bar{\gamma})}{\zeta_{i}(\bar{\mu})}\right)\right) \leq \frac{1}{2 \cdot M_{L}},
$$

where $\gamma:=\max \left\{\gamma_{1}, \gamma_{2}\right\}, \beta:=\min \left\{\alpha_{1}, \alpha_{2}\right\}$, and $C_{2}(\gamma)=\max \left\{c_{12}\left(\gamma_{1}\right), c_{22}\left(\gamma_{2}\right)\right\}$, then system (1) possesses at least one solution $\bar{x} \in C$ such that $\bar{\mu} \leq \bar{x}(t) \leq \bar{\gamma}, t \in[1, e]$.

Proof The proof follows the lines of the preceding theorem with some necessary changes. It will be rewritten in such a way to facilitate possible future extensions. Namely, in this case let us briefly describe some necessary changes. Fix any $\bar{x}=\left(x_{1}, x_{2}\right) \in C$. Operator $T$ is defined as follows:

$$
T_{1}(\bar{x})(t):=H_{1}(t, \bar{x}(t))+\frac{F_{1}\left(x_{2}\right)(t)}{\Gamma\left(\alpha_{1}\right)} \int_{1}^{t}\left(\log \frac{t}{s}\right)^{\alpha_{1}-1} f_{1}(s, \bar{x}(s)) \frac{\eta_{1}^{\star}(\bar{x}(s))}{\zeta_{1}^{\star}(\bar{x}(s))} \frac{d s}{s}, \quad t \in[1, e]
$$

and

$$
T_{2}(\bar{x})(t):=H_{2}(t, \bar{x}(t))+\frac{F_{2}\left(x_{1}\right)(t)}{\Gamma\left(\alpha_{2}\right)} \int_{1}^{t}\left(\log \frac{t}{s}\right)^{\alpha_{2}-1} f_{2}(s, \bar{x}(s)) \frac{\eta_{2}^{\star}(\bar{x}(s))}{\zeta_{2}^{\star}(\bar{x}(s))} \frac{d s}{s}, \quad t \in[1, e], \quad(20)
$$

so we define the set $Q$ as a subset of $C[1, e]$ consisting of positive functions having, for any $r>0$, the following modulus of equicontinuity estimated by

$$
\omega(Q, r)=C_{2}(\gamma) \cdot \omega(b, r)+\frac{\tilde{\Psi}(|t-s|)}{1-K^{*}}
$$

but where a constant $K^{*}$ is defined as

$$
K^{*}:=\left(C_{2}(\gamma) \cdot M_{L}+\frac{2 \cdot M_{L} \cdot \tilde{\Psi}(1) \max _{i}\left\|M_{i}\right\|_{\psi}}{\Gamma(\alpha)} \cdot \max _{i}\left\{\frac{\eta_{i}(\bar{\gamma})}{\zeta_{i}(\bar{\mu})}\right\}\right)<\frac{1}{2} .
$$

Let us recall that in the proof of Theorem 2 we had $\omega(Q,|t-s|)=\left[\frac{B(t, s)+K \tilde{\Psi}(|t-s|)}{1-K^{*}}\right]$.

To show that $T: Q \rightarrow Q$, we need to infer that the modulus of continuity $\omega(Q)$ is preserved by $T$. Recall that if $x$ and $y$ are functions with moduli respectively $\omega_{1}$ and $\omega_{2}$, then any linear combination $a x+b y$ has the modulus of continuity $|a| \omega_{1}+|b| \omega_{2}$. Then, with no 
essential changes in (Step 1), by applying Proposition 2 , for any $i=1,2$, we get an estimation:

$$
\begin{aligned}
\left|T_{i}\left(x_{i}\right)\left(t_{2}\right)-T_{i}\left(x_{i}\right)\left(t_{1}\right)\right| \leq & C_{1}(\gamma)\left|b\left(t_{2}\right)-b\left(t_{1}\right)\right|+C_{2}(\gamma) \cdot M_{L} \cdot \omega\left(Q,\left|t_{2}-t_{1}\right|\right) \\
& +\frac{M_{L} \cdot \omega\left(Q,\left|t_{2}-t_{1}\right|\right)}{\Gamma(\alpha)} \max _{i}\left(\frac{\eta_{i}(\bar{\gamma})}{\zeta_{i}(\bar{\mu})}\right) \cdot 2 \tilde{\Psi}(1) \max _{i}\left\|M_{i}\right\|_{\psi} \\
& +\frac{4 \gamma}{\Gamma(\alpha)} \max _{i}\left(\frac{\eta_{i}(\bar{\gamma})}{\zeta_{i}(\bar{\mu})}\right) \cdot \tilde{\Psi}\left(\left|t_{2}-t_{1}\right|\right) \max _{i}\left\|M_{i}\right\|_{\psi},
\end{aligned}
$$

and then

$$
\left|T_{i}\left(x_{i}\right)\left(t_{2}\right)-T_{i}\left(x_{i}\right)\left(t_{1}\right)\right| \leq \omega\left(Q,\left|t_{2}-t_{1}\right|\right),
$$

so $T: Q \rightarrow Q$.

In (Step 2) we follow the lines of the proof of Theorem 2, with only minor changes, together with Proposition 2. For any sequence $\bar{x}_{n} \rightarrow \bar{x}$ in $Q \cap\left(\bar{Q}_{2} \backslash Q_{1}\right)$, i.e., $\bar{x}_{n}(t)=$ $\left(x_{1, n}(t), x_{2, n}(t)\right) \rightarrow \bar{x}(t)=\left(x_{1}(t), x_{2}(t)\right)$ uniformly in $C$, we get

$$
\begin{aligned}
\left|T_{i}\left(\bar{x}_{n}\right)(t)-T_{i}(\bar{x})(t)\right| \leq & C_{2}(\gamma) \cdot\left\|\bar{x}_{n}(t)-\bar{x}(t)\right\|_{\mathbb{R}^{2}} \\
& +\left|F_{i}\left(\bar{x}_{n}\right)(t)-F_{i}(\bar{x})(t)\right| \cdot \frac{2 \tilde{\Psi}(1) \max _{i}\left\|M_{i}\right\|_{\psi}}{\Gamma(\alpha)} \cdot \max _{i}\left\{\frac{\eta_{i}(\bar{\gamma})}{\zeta_{i}(\bar{\mu})}\right\} \\
& +\frac{\gamma}{\Gamma(\alpha)} \max _{i}\left(\frac{\eta_{i}(\bar{\gamma})}{\zeta_{i}(\bar{\mu})} \int_{1}^{t}\left(\log \frac{t}{s}\right)^{\alpha-1}\left|f_{i}\left(s, \bar{x}_{n}(s)\right)-f_{i}(s, \bar{x}(s))\right| \frac{d s}{s}\right) \\
& +\frac{2 \gamma \tilde{\Psi}(1) \max _{i}\left\|M_{i}\right\|_{\psi}}{\Gamma(\alpha)} \max _{i}\left(\max _{t \in[1, e]}\left|\frac{\eta_{i}^{\star}\left(\bar{x}_{n}(t)\right)}{\zeta_{i}^{\star}\left(\bar{x}_{n}(t)\right)}-\frac{\eta_{i}^{\star}(\bar{x}(t))}{\zeta_{i}^{\star}(\bar{x}(t))}\right|\right) .
\end{aligned}
$$

Thus we obtain the continuity of $T$ (some changes are results of the presence of constant $M_{L}$, so please let us omit the details).

We should note that in (Step 3) of Theorem 2 we proved two facts that $T$ maps bounded and equicontinuous sets into the same family of sets with $T: Q \rightarrow Q$. Thus we need to prove the same property here (see the new definition of $Q$ ). The boundedness of $T(Q)$ is proved in the same manner. Note that the last steps of the proof need not be modified.

We need to mention that the case of Lipschitz mappings forms the simplest case in which (17) holds true.

Remark 2 Let us indicate one more version of our result, which can be useful for some spaces other than $C[1, e]$, i.e., with discontinuous solutions. A typical one is the space of regulated functions $X=G([1, e])$, i.e., the Banach space of functions having finite one-side limits at every point (but possibly discontinuous) equipped with the sup-norm or the space of left (or: right) continuous functions (see [11] for more details about this space and some quadratic problems in this space). For example, it means that our results could be used for impulsive or other problems having discontinuous solutions, too.

Proposition 3 Let $\psi$ be a Young function satisfying (3). Assume that a function space $X \subset L_{\psi}([1, e])$ is such that its space of pointwise multipliers $M_{p}(X)$ contains the space 
$C[1, e]$ and that $F\left(x_{1}, x_{2}\right)=\left(F_{1}\left(x_{2}\right), F_{2}\left(x_{1}\right)\right): X \times X \rightarrow X \times X$ with the norm $\left\|\left(x_{1}, x_{2}\right)\right\|_{C}=$ $\max \left\{\left\|x_{1}\right\|_{X},\left\|x_{2}\right\|_{X}\right\}$ is bounded and continuous. If the space $X$ is equipped with the supnorm $\|x\|_{X}=\sup _{t \in[1, e]}|x(t)|$, then under the assumptions of Theorem 2, system (1) possesses at least one solution $\bar{x} \in X$ such that $\bar{\mu} \leq \bar{x}(t) \leq \bar{\gamma}, t \in[1, e]$.

As $X \subset L_{\psi}([1, e])$, then applying Lemma 1 in its generality, we ensure that the fractional operator is well defined on $X$. The remaining part of the proof runs as in the main theorem. Clearly, in this case instead of continuity of $H$, it suffices to assume that $H(\cdot, \bar{x}(\cdot)) \in X$.

Corollary 1 Under the assumptions of Theorem 2 with $C([1, e])$ respectively replaced by $G([1, e])$, system $(1)$ possesses at least one regulated positive solution $\bar{x} \in G([1, e]) \times G([1, e])$ such that $\bar{\mu} \leq \bar{x}(t) \leq \bar{\gamma}, t \in[1, e]$.

We close this section by introducing the following examples, which illustrate the results proved in Theorem 2 and Proposition 2 (cf. also [9]). We start with the following one.

Example 3.1 Consider the singular system of cubic equations of type (15), i.e., for Proposition 2:

$$
\begin{aligned}
x_{1}(t)= & \frac{1+t}{20}+\log t \frac{x_{2}^{2}(t)}{8} \\
& +\frac{x_{2}^{2}(t)}{\Gamma(1 / 2)} \int_{1}^{t}\left(\log \frac{t}{s}\right)^{-1 / 2}\left[\frac{\left(x_{1}(s)+x_{2}(s)\right)^{x_{1}(s)+x_{2}(s)}}{e^{20\left(x_{1}(s)+x_{2}(s)\right)}-1}\right] \log \left(s-1+\left|x_{2}(s)\right|\right) \frac{d s}{s}, \\
& t \in[1, e], \\
x_{2}(t)= & \frac{t}{10}+(t-1) \frac{\left(x_{1}(t)+x_{2}(t)\right)}{15} \\
& +\frac{x_{1}^{2}(t)}{6 \Gamma(1 / 2)} \int_{1}^{t}\left(\log \frac{t}{s}\right)^{-1 / 2}\left[\frac{\log \left(0.1+x_{1}(s)+x_{2}(s)\right)}{\sqrt{x_{1}(s)+x_{2}(s)}}\right] \log \left(\frac{e-1}{e-s}\right) e^{\frac{x_{1}}{16}} \frac{d s}{s}, \\
& t \in[1, e] .
\end{aligned}
$$

Observe that (21) is a special case of (15) if we put $\alpha_{1}=\alpha_{2}=\frac{1}{2}, F\left(x_{1}, x_{2}\right)=\left(x_{2}^{2}, x_{1}^{2}\right)$ and

$$
\left\{\begin{array} { l } 
{ H _ { 1 } ( t , \overline { x } ) = \frac { 1 + t } { 2 0 } + \frac { x _ { 2 } ^ { 2 } } { 8 } \operatorname { l o g } t , } \\
{ f _ { 1 } ( t , \overline { x } ) = \operatorname { l o g } ( t - 1 + | x _ { 2 } | ) , } \\
{ \eta _ { 1 } ( \overline { x } ) = ( x _ { 1 } + x _ { 2 } ) ^ { x _ { 1 } + x _ { 2 } } , } \\
{ \zeta _ { 1 } ( \overline { x } ) = e ^ { 2 0 ( x _ { 1 } + x _ { 2 } ) } - 1 }
\end{array} \quad \text { and } \quad \left\{\begin{array}{l}
H_{2}(t, \bar{x})=\frac{t}{10}+\frac{\left(x_{1}+x_{2}\right)}{15}(t-1), \\
f_{2}(t, \bar{x})=e^{\frac{x_{1}}{16}} \log \left(\frac{e-1}{e-t}\right), \\
\eta_{2}(\bar{x})=\log \left(0.1+x_{1}+x_{2}\right), \\
\zeta_{2}(\bar{x})=6 \sqrt{x_{1}+x_{2}} .
\end{array}\right.\right.
$$

In what follows, we show that the functions involved in system (21) satisfy inequality (18) from Theorem 2 . To do this, choose $\bar{\mu}=(0.1,0.1)$ and $\bar{\gamma}=(0.4,0.6)$. Then, for every $\bar{x} \leq$ $(0.1,0.1)$ and every $t \in[1, e]$, we have

$$
H_{1}(t, x)>0.1, \quad H_{2}(t, x)>0.1 \quad \text { and then } \quad 2 H_{1}(t, 0.1)<0.4, \quad 2 H_{2}(t, 0.1)<0.6
$$

On the other hand, for every $t, s \in[1, e]$ and $\bar{x}, \bar{y} \in \mathbb{R}^{2}$, we have

$$
\left|H_{1}(t, \bar{x})-H_{1}(s, \bar{y})\right| \leq \frac{1}{20}|t-s|+\frac{\log t}{8}\left|x_{2}^{2}-y_{2}^{2}\right|+\frac{y_{2}^{2}}{8}|\log t-\log s| .
$$


By using the classical mean value theorem, we have

$$
\begin{aligned}
\left|H_{1}(t, \bar{x})-H_{1}(s, \bar{y})\right| & \leq \frac{1}{20}|t-s|+\frac{\log t\left|x_{2}+y_{2}\right|}{8}\left|x_{2}-y_{2}\right|+\frac{\gamma_{2}^{2}}{8 \varsigma}|t-s| \\
& \leq\left(\frac{1}{20}+\frac{\gamma_{2}^{2}}{8}\right)|t-s|+\frac{2 \gamma_{2}}{8}\|\bar{x}-\bar{y}\|_{\mathbb{R}^{2}}, \quad \varsigma \in(1, e) .
\end{aligned}
$$

Obviously, $H_{1}$ satisfies modified assumption (4.) from Proposition 2 with $\mu_{1}=0.1, \gamma_{1}=$ $\left.0.4, b(t)=t, t \in[1, e], c_{12}(\gamma)=0.15, \eta_{1}(\bar{\gamma})=\left(\gamma_{1}+\gamma_{2}\right)^{\gamma_{1}+\gamma_{2}}=1, \eta_{1}(\bar{\mu})=e^{20\left(\mu_{1}+\mu_{2}\right)}-1\right)=e^{4}-1$, and $f_{1}(t, \bar{x})=\log \left(t-1+\left|x_{2}\right|\right)$.

Note that, for any $r>0$, we have $\omega(F(\bar{x}), r)=\omega\left(\bar{x}^{2}, r\right)=2\|\bar{x}\|_{\mathbb{R}^{2}} \cdot \omega(\bar{x}, r) \leq 2 \gamma \omega(\bar{x}, r)$. Thus $\omega\left(F\left(B_{\gamma}\right)\right) \leq 2 \gamma \omega\left(B_{\gamma}\right)$. It implies that $T$ maps equicontinuous subsets of $Q \cap\left(\bar{Q}_{2} \backslash Q_{1}\right)$ into the sets with the same property, so the assertion of (Step 3) in the proof of both Theorem 2 and Proposition 2 is satisfied (assumption (17) holds true with $M_{L}=2 \gamma$ ).

Put $\psi(u)=e^{|u|}-|u|-1$. Let us recall, as claimed in [16, Example 1], that it is an example of Young functions satisfying (3) for any $\alpha \in(0,1)$.

Hence $\left.M_{1}(t)=\max \left\{\log \left(t-1+\gamma_{2}\right),-\log (t-1)\right\}\right)$ and $\left\|M_{1}\right\|_{\psi} \leq 2.2$. Owing to this, the definition of $\tilde{\Psi}(1) \leq 3$ holds for any $\alpha \in(0,1)$. Similarly, we have

$$
\begin{aligned}
\left|H_{2}(t, \bar{x})-H_{2}(s, \bar{y})\right| & \leq \frac{1}{10}|t-s|+\frac{(t-1)}{15}\|\bar{x}-\bar{y}\|_{\mathbb{R}^{2}}+\frac{y_{1}+y_{2}}{15}|t-s| \\
& \leq\left(\frac{1}{10}+\frac{\gamma_{1}+\gamma_{2}}{15}\right)|t-s|+\frac{e-1}{15}\|\bar{x}-\bar{y}\|_{\mathbb{R}^{2}} .
\end{aligned}
$$

Thus $H_{2}$ satisfies assumption (4.) with $\mu_{2}=0.1, \gamma_{2}=0.6, b(t)=t, t \in[1, e], c_{22}(\gamma)=$ $0.11, \eta_{2}(\bar{\gamma})=\log \left(0.1+\gamma_{1}+\gamma_{2}\right)=\log 1.1$ and $\zeta_{2}(\bar{\mu})=6 \sqrt{\mu_{1}+\mu_{2}}=6 \sqrt{0.2}$. and $f_{2}(t, \bar{x})=$ $e^{\frac{x_{1}(t)}{16}} \log \left(\frac{e-1}{e-t}\right)$ (hence $\left.M_{2}(t)=e^{\frac{\gamma_{1}}{16}} \log \left(\frac{e-1}{e-t}\right)\right)$.

By [16, Example 1], we get $\left\|M_{2}\right\|_{\psi} \leq 2 e^{\frac{\gamma_{2}}{16}}$. Therefore,

$$
2\left(C_{2}(\gamma)+\frac{4 \cdot \gamma \cdot \tilde{\Psi}(1)\left\|M_{i}\right\|_{\psi}}{\Gamma(1 / 2)} \frac{\log 1.1}{6 \sqrt{0.2}}\right) \leq 1
$$

Thus, the hypotheses of Theorem 2 and Proposition 2 are satisfied, hence we conclude that problem (21) has at least one continuous solution $\bar{x}$ such that $0.1 \leq\|\bar{x}\|_{\mathbb{R}^{2}} \leq 0.6, t \in[1, e]$.

We give an example showing that inequality (18) is necessary for the proof of Theorem 2. In other words, the following example is useful in proving that the hypothesis involved in inequality (18) is really essential for the existence of continuous solutions and cannot be eliminated.

Example 3.2 Consider the system

$$
\begin{array}{ll}
x_{1}(t)=1+\frac{x_{2}(t)}{\Gamma(0.5)} \int_{1}^{t}\left(\log \frac{t}{s}\right)^{-0.5} \frac{d s}{s}, & t \in[1, e], \\
x_{2}(t)=1+\frac{x_{1}(t)}{\Gamma(0.5)} \int_{1}^{t}\left(\log \frac{t}{s}\right)^{-0.5} \frac{d s}{s}, & t \in[1, e] .
\end{array}
$$

Observe that system (22) is a particular case of (1) if we put $[a, b]=[1, e], \alpha_{1}=\alpha_{2}=\frac{1}{2}$, and $f_{i}(t, \bar{x})=H_{i}(t, \bar{x})=\eta_{i}(\bar{x})=\zeta_{i}(\bar{x})=1, i=1,2$. In this case, we deduce that $C_{2}(\gamma)=0$ and 
$\left\|M_{i}\right\|_{\psi_{3}}=\sqrt[3]{\frac{e-1}{3}}$, where $\psi_{3}=\frac{|u|^{3}}{3}$ (by the definition of $\psi$, we get $\tilde{\Psi}(1) \approx 1.9$ ). In what follows, we show that our choice of the functions involved in system (22) does not satisfy inequality (18). Meanwhile, we will show that system (22) admits no positive continuous solutions on $[1, e]$. Evidently, on the one hand, we have

$$
\left(C_{2}(\gamma)+\frac{2 \tilde{\Psi}(1) \max _{i}\left\|M_{i}\right\|_{\psi}}{\Gamma(\alpha)} \max _{i}\left(\frac{\eta_{i}(\bar{\gamma})}{\zeta_{i}(\bar{\mu})}\right)\right) \simeq \frac{3.8}{\sqrt{\pi}} \sqrt[3]{\frac{e-1}{3}}>\frac{1}{2} .
$$

From which, it can be easily seen that there are no positive constants $\bar{\mu} \leq(1,1)$ and $\bar{\gamma} \geq(2,2)$ such that inequality $(18)$ holds.

On the other hand, system (22) has no positive continuous solutions on $[1, e]$. To see this, we note that (22) is equivalent to the following estimate:

$$
\begin{aligned}
& x_{1}(t)=1+\frac{x_{2}(t)}{\Gamma(1.5)}(\log t)^{0.5}, \\
& x_{2}(t)=1+\frac{x_{1}(t)}{\Gamma(1.5)}(\log t)^{0.5}, \quad t \in[1, e] .
\end{aligned}
$$

Thus, the unique solution of (22) would be given by

$$
x_{i}(t)=\frac{1}{1-\frac{(\log t)^{0.5}}{\Gamma(1.5)}}<0, \quad \forall t>e^{\frac{\pi}{4}}, i=1,2,
$$

which is not positive on $[1, e]$. It is precisely what one would expect. It points out the fact that the assertion of Theorem 2 is no longer true if we remove the hypothesis involved in inequality (18).

\section{An application to integral equations}

We need to show that the singularity of system (1) allows us to investigate the problem of the existence of a continuous solution for some nonlinear Hadamard fractional integral equations. It will be done after proving a preliminary proposition.

In what follows, we let $\phi:(0, \infty] \rightarrow \mathbb{R}^{+}$be a continuous function such that the map

$$
(x, y) \rightarrow y^{p} \phi(x / y) \text { is nondecreasing with respect to the ordering in } \mathbb{R}^{2} .
$$

Let us present a simple sufficient condition for (23) as follows.

Proposition 4 Let $p>0$. If the function $\phi$ is continuous nondecreasing on $(0, \infty]$ with the property that $z \rightarrow z^{-p} \phi(z)$ is nonincreasing on $\mathbb{R}^{+}$, then (23) holds true.

Proof Obviously (23) is equivalent to the assertion that the map (23) is nondecreasing with respect to each variable separately. In other words, it is necessary and sufficient that $f_{c}(z):=\phi(c z)$ and $g_{c}(z):=z^{p} \phi(c / z)$ are both nondecreasing on $\mathbb{R}^{+}$for every $c>0$.

Of course, a simpler equivalent condition is that $z \rightarrow \phi(z)$ and $z \rightarrow z^{p} \phi(1 / z)$ are both nondecreasing $\mathbb{R}^{+}$. Noting that $(\cdot)^{p} \phi(1 / \cdot)$ is nondecreasing if and only if $z \rightarrow z^{-p} \phi(z)$ is nonincreasing on $\mathbb{R}^{+}$, one can reformulate our condition now equivalently as follows: $\phi: \mathbb{R}^{+} \rightarrow \mathbb{R}^{+}$is any nondecreasing function with the property that $z \rightarrow z^{-p} \phi(z)$ is nonincreasing $\mathbb{R}^{+}$, which is our expected thesis. 


\section{Remark 3}

1. For $z>1$, there must hold $z^{-p} \phi(z) \leq \phi(1)$ and then $\phi(z) \leq M z^{p}$, i.e., $\phi$ cannot grow faster than constant times $z^{p}$.

2. For any $p>0$, there are a lot of functions $\phi$ with the required property, because the factor $z \rightarrow z^{-p}$ is strictly decreasing. For instance, let $\phi(z):=1+z^{2}, p \geq 2$.

Moreover, one can define $\phi$ piecewise on $(0, a]$ and $[a, \infty)$ by choosing $\phi$ on the first interval such that the monotonicity conditions are satisfied on this first interval and then extending $\phi$ (without changing $\phi(a)$ ) such that the monotonicity conditions are satisfied on the second interval (e.g., constant or some other polynomial). Of course, one could also choose more than two intervals.

The simplest example is to define $\phi$ piecewise polynomials: choose some numbers $r_{1}, r_{2}, \ldots, r_{\infty} \in[0, p)$ (a finite or countable set) and a corresponding partition $0=s_{0}<s_{1}<s_{2}<\cdots<s_{\infty}=\infty$ of $[0, \infty)$ and put

$$
\phi(z)=\phi_{i}(z)+c_{i}, \quad \text { for } z \in\left[s_{i-i}, s_{i}\right),(i=1,2, \ldots, \infty),
$$

where $\phi_{i}(z)=z^{r_{i}}, c_{0}$ is arbitrary and $c_{i+1}=c_{i}+\phi_{i}\left(s_{i}\right)$.

We can generalize this construction for functions which are not even "piecewise" power functions $\phi_{i}$.

For instance, for $r \in[0, p], w>0$, and real $a>0$, the function

$$
h(z):=t^{r}(\log (z-a+e))^{w}
$$

is nondecreasing on $(a, \infty)$ with the property that $H(t)=z^{-p} h(z)$ satisfies $H^{\prime}(z)=z^{r-p-1} G(z)$, where

$$
G(z):=(r-p)(\log (z-a+e))^{w}+w(\log (z-a+e))^{w-1} /(z-a+e) .
$$

Since $r-p<0$, the first summand of $G$ is negative and the second is convergent to 0 as $z \rightarrow \infty$. Therefore, there is a constant $C$ (depending on $p, r, w, a$ ) such that $G(z) \leq 0$ for $t \geq C$ (we can choose $C$ large enough). As $C \geq a$, this assertion makes sense.

So, if $s_{i} \geq s_{i-1} \geq C$, we can also choose $\phi_{i}(z)=h(z)-h\left(s_{i-1}\right)$. Of course, different values for the constants $r, a, w$ are possible on different intervals. Moreover, instead of $h$ we can start with a lot of different functions. However, the class of continuous functions, which satisfies the required growth conditions, is really huge.

Example 4.1 We give one more direct example of a function $\phi$ satisfying (23). Let $p=2$ and define the continuous nondecreasing function $\phi:(0, \infty] \rightarrow[0, \infty]$ by $\phi(z):=z \log (1+z)$. Obviously, the function $\phi(z):=z^{-2} \phi(z)$ satisfies $\phi^{\prime}(z)=z^{-2} G(z)$, where

$$
G(z)=\frac{z}{1+z}-\log (1+z)
$$

The mean value theorem on $G$ on the interval $[0, z]$ yields $G(z)<G(0)=0$ for every $z>0$. Thus the map $z \rightarrow z^{-2} \phi(z)$ is decreasing, and so $\phi$ satisfies (23) as required. 
Clearly, when studying nonquadratic problems, it is sufficient to follow some ideas from Theorem 2. Let us present now the following version for nonquadratic singular system of fractional integral equations with Hadamard integrals.

Theorem 3 Let $h_{1}, h_{2} \in C[[a, b],(0, \infty)]$ and $\phi_{i}:(0, \infty] \rightarrow \mathbb{R}^{+}(i=1,2)$ be continuous functions satisfying (23) with $p_{i}>0(i=1,2)$. Suppose that there is $0<\mu_{i}<\gamma_{i}(i=1,2)$ such that, for any $t \in[a, b], \bar{\mu} \leq\left(h_{1}(t) x_{2}, h_{2}(t) x_{1}\right)$ holds true for every $\bar{x} \leq \bar{\mu}$ and $\bar{\gamma} \geq$ $\left(2\left\|h_{1}\right\| \mu_{2}, 2\left\|h_{2}\right\| \mu_{1}\right)$. If

$$
\left(\max _{i}\left\|h_{i}\right\|+\frac{2 \tilde{\Psi}(1) \max _{i}\left\|M_{i}\right\|_{\psi}\left(\frac{\gamma}{\mu}\right)^{\max _{i}\left\{P_{i}\right\}}}{\Gamma(\alpha)} \max \left\{\phi_{1}\left(\frac{\gamma_{2}}{\gamma_{1}}\right), \phi_{2}\left(\frac{\gamma_{1}}{\gamma_{2}}\right)\right\}\right) \leq \frac{1}{2},
$$

then the following singular system of Hadamard fractional integral equations

$$
\begin{aligned}
& y_{1}(t)=h_{1}(t)+\mathfrak{J}_{1}^{\alpha_{1}} M_{1}(t) \frac{\phi_{1}\left(y_{2}(t)\right)}{\left(y_{2}(t)\right)^{p_{1}}}, \quad t \in[1, e], \alpha_{1}>0, \\
& y_{2}(t)=h_{2}(t)+\mathfrak{J}_{1}^{\alpha_{2}} M_{2}(t) \frac{\phi_{1}\left(y_{1}(t)\right)}{\left(y_{1}(t)\right)^{p_{2}}}, \quad t \in[1, e], \alpha_{2}>0,
\end{aligned}
$$

admits at least one solution $\bar{y}=\left(y_{1}, y_{2}\right) \in C$ such that $\left(\frac{\mu}{\gamma}, \frac{\mu}{\gamma}\right) \leq \bar{y}(t) \leq\left(\frac{\gamma}{\mu}, \frac{\gamma}{\mu}\right), t \in[1, e]$ and that $y_{1}(\cdot) y_{2}(\cdot)=1$.

Proof Consider system (1) with the following parameter functions:

$$
\left\{\begin{array} { l } 
{ H _ { 1 } ( t , \overline { x } ) = h _ { 1 } ( t ) x _ { 2 } , } \\
{ f _ { 1 } ( t , \overline { x } ) = M _ { 1 } ( t ) x _ { 1 } , } \\
{ \eta _ { 1 } ( x _ { 1 } , x _ { 2 } ) = x _ { 1 } ^ { p _ { 1 } - 1 } \phi _ { 1 } ( x _ { 2 } / x _ { 1 } ) , } \\
{ \zeta _ { 1 } ( \overline { x } ) = x _ { 2 } ^ { p _ { 1 } } }
\end{array} \quad \text { and } \quad \left\{\begin{array}{l}
H_{2}(t, \bar{x})=h_{2}(t) x_{1}, \\
f_{2}(t, \bar{x})=M_{2}(t) x_{2}, \\
\eta_{2}(\bar{x})=x_{2}^{p_{2}-1} \phi_{1}\left(x_{1} / x_{2}\right), \\
\zeta_{2}(\bar{x})=x_{1}^{p_{2}} .
\end{array}\right.\right.
$$

In this case, in view of (24), one can verify that the assumptions of Theorem 2 are satisfied. Therefore, the system

$$
\begin{cases}x_{1}(t)=h_{1}(t) x_{2}(t)+x_{2}(t) \mathfrak{J}_{1}^{\alpha_{1}} M_{1}(t) \frac{\left(x_{1}(t)\right)^{p} \phi_{1}\left(x_{2}(t) / x_{1}(t)\right)}{\left(x_{2}(t) p^{p 1}\right.}, & t \in[1, e], \alpha_{1}>0, \\ x_{2}(t)=h_{2}(t) x_{1}(t)+x_{1}(t) \mathfrak{J}_{1}^{\alpha_{2}} M_{2}(t) \frac{\left(x_{2}(t)\right)^{p} \phi_{1}\left(x_{1}(t) / x_{2}(t)\right)}{\left(x_{1}(t)\right)^{p 2}}, & t \in[1, e], \alpha_{2}>0\end{cases}
$$

has at least one solution $\bar{x} \in C$ such that $(\mu, \mu) \leq \bar{x}(t) \leq(\gamma, \gamma), t \in[1, e]$. Since $\bar{x} \neq(0,0)$, it can be proved that system (26) is equivalent to the following one:

$$
\left\{\begin{array}{l}
\frac{x_{1}(t)}{x_{2}(t)}=h_{1}(t)+\mathfrak{J}_{1}^{\alpha_{1}} M_{1}(t)\left(\frac{x_{1}(t)}{x_{2}(t)}\right)^{p_{1}} \phi_{1}\left(x_{2}(t) / x_{1}(t)\right) \\
\frac{x_{2}(t)}{x_{1}(t)}=h_{2}(t)+\mathfrak{J}_{1}^{\alpha_{2}} M_{2}(t)\left(\frac{x_{2}(t)}{x_{1}(t)}\right)^{p_{2}} \phi_{2}\left(x_{1}(t) / x_{2}(t)\right)
\end{array}\right.
$$

Now, put $y_{1}=x_{1} / x_{2}$ and $y_{2}=x_{2} / x_{1}$, it follows that system (25) has at least one solution $\bar{y}=\left(y_{1}, y_{2}\right) \in C$ such that $\left(\frac{\mu}{\gamma}, \frac{\mu}{\gamma}\right) \leq \bar{y}(t) \leq\left(\frac{\gamma}{\mu}, \frac{\gamma}{\mu}\right), t \in[1, e]$ and that $y_{1}(\cdot) y_{2}(\cdot)=1$.

Finally, we present an example to show the applicability of the preceding result. 
Example 4.2 Consider the following singular system of fractional order integral equations:

$$
\begin{aligned}
& y_{1}(t)=\frac{1}{10}+\mathfrak{J}_{1}^{0.5} \frac{1+y_{2}^{2}(t)}{15 y_{2}^{2}(t)}, \quad t \in[1, e], \\
& y_{2}(t)=\frac{1}{10}+\mathfrak{J}_{1}^{0.5} \frac{\log \left(1+y_{1}(t)\right)}{5 y_{1}(t)}, \quad t \in[1, e] .
\end{aligned}
$$

Observe that the above system is a special case of system (25). Namely, if we put $M_{i}(t)=1$ (with $M_{i}(t) \in L_{\psi_{3}}[1, e]$ and $\psi(u)=\frac{|u|^{3}}{3}$ ), $\tilde{\Psi}(1)<2,\left\|M_{i}\right\|_{\psi_{3}}=\sqrt[3]{\frac{e-1}{3}}$ and

$$
\phi_{1}(z)=\frac{1+z^{2}}{15}, \quad \phi_{2}(z)=\frac{z}{5} \log (1+z), p_{i}=2, \quad z \in \mathbb{R}^{+} .
$$

It is already seen that (cf. Example 4.1) the functions involved in (27) satisfy the assumptions of Theorem 3 with $\alpha_{1}=\alpha_{2}=0.5$.

Evidently, if we choose $\mu_{1}=\mu_{2}=\mu \leq \frac{1}{10}$ and $\gamma_{1}=\gamma_{2}=\gamma=\frac{11}{10} \mu$, one can get

$$
\begin{gathered}
\max _{i}\left\|h_{i}\right\|+\frac{2 \tilde{\Psi}(1) \max _{i}\left\|M_{i}\right\|_{\psi}\left(\frac{\gamma}{\mu}\right)^{\max _{i}\left\{P_{i}\right\}}}{\Gamma(\alpha)} \max \left\{\phi_{1}\left(\frac{\gamma_{2}}{\gamma_{1}}\right), \phi_{2}\left(\frac{\gamma_{1}}{\gamma_{2}}\right)\right\} \\
\leq\left(\frac{1}{10}+\left(\frac{11}{10}\right)^{2} \frac{4}{\sqrt{\pi}} \sqrt[3]{\frac{e-1}{3}} \max \left\{\phi_{1}(1), \phi_{2}(1)\right\}\right)<1 / 2 .
\end{gathered}
$$

Thus, inequality (24) holds true. Consequently, in view of Theorem 3, system (27) has at least one solution $\bar{y} \in C$ such that $\left(\frac{10}{11}, \frac{10}{11}\right) \leq \bar{y}(t) \leq\left(\frac{11}{10}, \frac{11}{10}\right), t \in[1, e]$ and that $y_{1}(\cdot) y_{2}(\cdot)=1$.

\section{Acknowledgements}

Not applicable.

Funding

Not applicable.

Availability of data and materials

Data sharing not applicable to this article as no datasets were generated or analysed during the current study.

Competing interests

The authors declare that they have no competing interests.

Authors' contributions

All authors contributed almost equally to the writing of this paper. All authors read and approved the final manuscript.

\section{Author details}

'Department of Mathematics and Computer Science, Faculty of Sciences, Alexandria University, Alexandria, Egypt.

${ }^{2}$ Institute of Mathematics, Faculty of Control, Robotics and Electrical Engineering, Poznan University of Technology,

Poznań, Poland.

\section{Publisher's Note}

Springer Nature remains neutral with regard to jurisdictional claims in published maps and institutional affiliations.

Received: 6 February 2020 Accepted: 17 May 2020 Published online: 05 June 2020

\section{References}

1. Ahmad, B., Ntouyas, S.K.: A fully Hadamard type integral boundary value problem of a coupled system of fractional differential equations. Fract. Calc. Appl. Anal. 17, 348-360 (2014)

2. Anastassiou, G.A.: Fractional Differentiation Inequalities. Springer, Berlin (2009)

3. Anastassiou, G.A.: Advances on Fractional Inequalities. Springer, Berlin (2011)

4. Argyros, I.K.: Quadratic equations and applications to Chandrasekhar's and related equations. Bull. Aust. Math. Soc. 32 , 275-292 (1985) 
5. Awad, H.K., Darwish, M.A., Metwali, M.: On a cubic integral equation of Urysohn type with linear perturbation of second kind. J. Math. Appl. 41, 29-38 (2018)

6. Banaś, J., Rzepka, B.: Monotonic solutions of a quadratic integral equation of fractional order. J. Math. Anal. Appl. 332 , 1370-1378 (2007)

7. Butzer, P.L., Kilbas, A.A., Trujillo, J.J.: Mellin transform analysis and integration by parts for Hadamard-type fractional integrals. J. Math. Anal. Appl. 270, 1-15 (2002)

8. Butzer, P.L., Kilbas, A.A., Trujillo, J.J.: Compositions of Hadamard-type fractional integration operators and the semigroup property. J. Math. Anal. Appl. 269, 387-400 (2002)

9. Caballero, J., O'Regan, D., Sadarangani, K.: On nondecreasing solutions of cubic integral equations of Urysohn type. Comment. Math. Prace Mat. 44, 39-53 (2004)

10. Carl, S., Heikkilä, S.: Fixed Point Theory in Ordered Sets and Applications: From Differential and Integral Equations to Game Theory. Springer, Berlin (2010)

11. Cichoń, K., Cichoń, M., Metwali, M.: On some parameters in the space of regulated functions and their applications. Carpath. J. Math. 34, 17-30 (2018)

12. Cichoń, M., Metwali, M.: On quadratic integral equations in Orlicz spaces. J. Math. Anal. Appl. 387, 419-432 (2012)

13. Cichoń, M., Metwali, M.: Existence of monotonic $L_{\varphi}$-solutions for quadratic Volterra functional-integral equations. Electron. J. Qual. Theory Differ. Equ. 2015(13), 1 (2015)

14. Cichoń, M., Metwali, M.: On a fixed point theorem for the product of operators. J. Fixed Point Theory Appl. 18, 753-770 (2016)

15. Cichoń, M., Metwali, M.: On the existence of solutions for quadratic integral equations in Orlicz spaces. Math. Slovaca 66, 1413-1426 (2016)

16. Cichoń, M., Salem, H.A.H.: On the solutions of Caputo-Hadamard Pettis-type fractional differential equations. Rev. R. Acad. Cienc. Exactas Fís. Nat., Ser. A Mat. 113, 3031-3053 (2019)

17. Darwish, M.: On Erdélyi-Kober fractional Urysohn-Volterra quadratic integral equations. Appl. Math. Comput. 273, 562-569 (2016)

18. Darwish, M., Sadarangani, K.: On a quadratic integral equation with supremum involving Erdélyi-Kober fractional order. Math. Nachr. 288, 566-576 (2015)

19. Dhage, B.C., Dhage, S.B., Graef, J.R.: Local attractivity and stability analysis of a nonlinear quadratic fractional integral equation. Appl. Anal. 95, 1989-2003 (2016)

20. Diethelm, K.: The Analysis of Fractional Differential Equations. Lecture Notes in Mathematics. Springer, Berlin (2004)

21. Ding, H.-S., Liu, M.-M., Nieto, J.J.: Multiple positive solutions for quadratic integral equations of fractional order. J. Funct. Spaces 2017, Article ID 4571067 (2017)

22. Guo, D., Lakshmikantham, V:: Nonlinear Problems in Abstract Cones. Notes and Reports in Mathematics in Science and Engineering, vol. 5. Academic Press, San Diego (2014)

23. Hadamard, J.: Essai sur l'étude des fonctions donnés par leur développment de Taylor. J. Math. Pures Appl. 8, 101-186 (1892)

24. Huang, H., Liu, W.: Positive solutions for a class of nonlinear Hadamard fractional differential equations with a parameter. Adv. Differ. Equ. 2018(1), 96 (2018)

25. Kilbas, A.A.: Hadamard-type fractional calculus. J. Korean Math. Soc. 38, 1191-1204 (2001)

26. Kilbas, A.A., Srivastava, H.M., Trujillo, J.J.: Theory and Applications of Fractional Differential Equations. Elsevier, Amsterdam (2006)

27. Kilbas, A.A., Titioura, A.A.: Nonlinear differential equations with Marchaud-Hadamard type fractional derivative in the weighted space of summable functions. Math. Model. Anal. 12, 343-356 (2007)

28. Metwali, M.: On a class of quadratic Urysohn-Hammerstein integral equations of mixed type and initial value problem of fractional order. Mediterr. J. Math. 13, 2691-2707 (2016)

29. Mishra, L.N., Sen, M.: On the concept of existence and local attractivity of solutions for some quadratic Volterra integral equation of fractional order. Appl. Math. Comput. 285, 174-183 (2016)

30. Salem, H.A.H.: On the existence of continuous solutions for a singular system of non-linear fractional integral equations. Appl. Math. Comput. 198, 443-452 (2008)

31. Salem, H.A.H.: On the quadratic integral equations and their applications. Comput. Math. Appl. 62, 2931-2943 (2011)

32. Salem, H.A.H.: Hadamard-type fractional calculus in Banach spaces. Rev. R. Acad. Cienc. Exactas Fís. Nat., Ser. A Mat. 113, 987-1006 (2018)

33. Salem, H.A.H.: On functions without pseudo derivatives having fractional pseudo derivatives. Quaest. Math. 42 1237-1252 (2019)

34. Salem, H.A.H., Cichon, M.: On solutions of fractional order boundary value problems with integral boundary conditions in Banach spaces. J. Funct. Spaces Appl. 2013, Article ID 428094 (2013)

35. Samko, S., Kilbas, A.A., Marichev, O.L.: Fractional Integrals and Derivatives. Gordon \& Breach, New York (1993)

36. Shammakh, W: A study of Caputo-Hadamard-type fractional differential equations with nonlocal boundary conditions. J. Funct. Spaces 2016, Article ID 7057910 (2016)

37. Zabreyko, P.P., Koshelev, A.I., Krasnosel'skii, M.A., Mikhlin, S.G., Rakovshchik, L.S., Ya, S.V. (eds.): Integral Equations-a Reference Text. Noordhoff, Leyden (1975) 